University of Warwick institutional repository: http://go.warwick.ac.uk/wrap This paper is made available online in accordance with publisher policies. Please scroll down to view the document itself. Please refer to the repository record for this item and our policy information available from the repository home page for further information.

To see the final version of this paper please visit the publisher's website. Access to the published version may require a subscription.

Author(s): ALAN ROBINSON and SARAH WHITEHOUSE Article Title: Operads and $\Gamma$-homology of commutative rings

Year of publication: 2002

Link to published version:

http://dx.doi.org/10.1017/S0305004102005534

Publisher statement: None 


\title{
Operads and $\Gamma$-homology of commutative rings
}

\author{
BY ALAN ROBINSON \\ Mathematics Institute, University of Warwick, Coventry CV4 7AL. \\ e-mail: car@maths.warwick.ac.uk \\ AND SARAH WHITEHOUSE $\dagger$ \\ Laboratoire de Géométrie-Algèbre, Université d'Artois - Pôle de Lens, \\ Rue Jean Souvraz, S.P. 18 - 62307 Lens, France. \\ e-mail: whitehouse@euler.univ-artois.fr
}

(Received 8 May 2000; revised 24 October 2000)

\begin{abstract}
We introduce $\Gamma$-homology, the natural homology theory for $E_{\infty}$-algebras, and a cyclic version of it. $\Gamma$-homology specializes to a new homology theory for discrete commutative rings, very different in general from André-Quillen homology. We prove its general properties, including flat base change and transitivity theorems. We give an explicit bicomplex for the $\Gamma$-homology of a discrete algebra, and elucidate connections with stable homotopy theory.
\end{abstract}

\section{Introduction}

In this paper, we construct and investigate a homology theory for coherently homotopy commutative $d g$-algebras, usually known as $E_{\infty}$-algebras. We call the theory $\Gamma$-homology because of its close connection with stable homotopy theory.

Since discrete commutative rings are $E_{\infty}$-rings, we obtain by specialization a new homology theory for commutative algebras. Just as André-Quillen homology is the appropriate homology for rings in algebraic geometry and commutative algebra, $\Gamma$-homology appears to be the homology of commutative rings which occurs in homotopy theory. For instance, the obstructions to an $E_{\infty}$ multiplication on a ring spectrum lie in the $\Gamma$-homology of the dual Steenrod algebra for that theory [19]. André-Quillen homology coincides with $\Gamma$-homology in characteristic zero (Corollary 6.6). In finite characteristic, the difference is well illustrated by the case of a polynomial algebra. As a commutative algebra this is free, so its André-Quillen homology is trivial (in positive degrees). As an $E_{\infty}$-algebra, it is far from free. Its $\Gamma$-homology is closely related to the homology of the Dyer-Lashof algebra, and is highly non-trivial (Theorem $4 \cdot 5$, Example $6 \cdot 7$ ).

$\Gamma$-homology for a discrete commutative algebra $B$ can perhaps best be understood by an analogy with Harrison homology. Harrison's theory was originally defined

$\dagger$ S.W. was supported by a TMR grant from the European Union, held at the Laboratoire d'Analyse, Géométrie et Applications (UMR 7539 au CNRS), Université Paris-Nord. A.R. was supported by the EU Homotopy Theory Network. 
as the homology of the quotient of the Hochschild complex by the subcomplex generated by nontrivial shuffle products. Equally, it is the homology of a complex obtained from Hochschild's by tensoring each term $B^{\otimes n}$ with a certain integral representation $V_{n}$ of the symmetric group $\Sigma_{n}$, and passing to $\Sigma_{n}$-covariants. This idea works well only in characteristic zero. A more satisfactory theory must necessarily involve the higher homology of the symmetric groups, as well as the covariants $H_{0}$. Our $\Gamma$-homology theory is constructed to do just that.

The definition of $\Gamma$-homology uses cyclic $E_{\infty}$ operads and a realization construction to make a homotopical version of the cotangent complex. The advantage of this approach, compared with relying upon resolutions, is that it allows us to construct a cyclic variant of $\Gamma$-homology. This seems to be a unique feature of our method, as no cyclic analogue of any variant of André-Quillen theory appears to be known. We shall have more to say about it elsewhere.

In the first three sections of the paper we cover these introductory topics, then prove flat base change and transitivity theorems for $\Gamma$-homology. We treat the $\Gamma$ homology of free $E_{\infty}$ algebras, and resolutions by these, in Section 4 . A close analogue of Miller's infinite-delooping spectral sequence [16] arises here.

In Section 5 we further justify our construction by showing that an $A_{\infty}$ (or 'permutation-free') analogue yields Hochschild homology and standard cyclic homology. The final two sections treat only discrete commutative algebras. In Section 6 we find an explicit bicomplex for their $\Gamma$-homology, prove the relationship with Kähler differentials and Exalcom, and show that the $\Gamma$-homology of an étale algebra is trivial. In the last section (Section 7) we use a method of Pirashvili and Richter to reconcile our definition with the elegant description which they recently published [17] and with one which we used in an earlier treatment [22].

The original motivation for the invention of $\Gamma$-homology was the classification of $E_{\infty}$ multiplicative structures on spectra in stable homotopy theory [19]. Some properties of the theory constructed by Robinson (unpublished) were proved in the thesis of Whitehouse [22]. The present paper is a general theory which has evolved over several years. Meanwhile related (though very different) work in nearby areas has been done by others. At an early stage Waldhausen envisaged a homology theory of our type as part of his 'brave new rings' programme. More recently Goerss and Hopkins [9-11] produced a categorical approach to $E_{\infty}$ structures on spectra. Using modern technology one can without great difficulty extend the definition of $\Gamma$-homology from $d g$-algebras to spectra, where it ought to be compared with the constructions made by Basterra [3] who gave a detailed treatment of unpublished ideas of Kriz. (The extension to spectra is analogous to extending Hochschild homology to topological Hochschild homology, which includes Mac Lane homology as a special case.)

\section{Operads, cyclic operads and cofibrancy}

We work in the category of $\mathbb{Z}$-graded chain complexes ( $d g$-modules) over a commutative ground ring $K$. (We might equally well have chosen simplicial modules.) Our principal definitions use Getzler and Kapranov's theory [7] of cyclic operads, but we require a non-unital version as in [8]. 


\subsection{Operads}

Let $\mathbb{S}$ denote the category of finite sets and isomorphisms of sets, $\mathbb{S}_{+}$the subcategory of non-empty sets, and $\mathbb{S}^{1}$ the category of based finite sets and isomorphisms. (Where necessary we assume without further mention that these have been replaced by equivalent small subcategories: our constructions do not depend upon the choice of these. One can for instance take just one set $\{1,2, \ldots, n\}$ in $\mathbb{S}$ for each $n \geqslant 0$, and similarly $\{0,1,2, \ldots, n\}$ for each $n \geqslant 0$ in $\mathbb{S}^{1}$, so that both categories become disjoint unions of symmetric groups.)

We are only interested in binary and higher-order operations. Therefore we define an operad $\mathscr{C}$ to have objects (which are chain complexes) $\mathscr{C}_{S}$ indexed by all finite sets $S$ with at least two elements; isomorphisms $\varphi_{*}: \mathscr{C}_{S} \rightarrow \mathscr{C}_{T}$ induced by isomorphisms $\varphi: S \rightarrow T$ of sets; and composition maps

$$
\circ_{t}: \mathscr{C}_{S} \otimes \mathscr{C}_{T} \longrightarrow \mathscr{C}_{S \sqcup_{t} T}
$$

for all nontrivial finite sets $S$ and $T$, and all elements $t \in T$, where $S \sqcup_{t} T$ is the deleted sum $S \sqcup(T \backslash\{t\})$. These data must satisfy standard conditions of functoriality and associativity of composition.

The induced isomorphisms give a left action of the symmetric group $\Sigma_{S}$ of automorphisms of $S$ on $\mathscr{C}_{S}$. One thinks of $\mathscr{C}_{S}$ as a parameter space of operations (in the sense of universal algebra) with inputs labelled by $S$, and a single output; the induced isomorphisms correspond to permutation of inputs, and the composition $o_{t}$ to substitution of the output of $\mathscr{C}_{S}$ for the input labelled $t$ in $\mathscr{C}_{T}$.

The operad $\mathscr{C}$ is said to be $E_{\infty}$ if for each $S$ the complex $\mathscr{C}_{S}$ is contractible and $\Sigma_{S}$-free. It is obviously sufficient to check this for $S=\{1,2, \ldots, n\}$, for all $n \geqslant 2$.

The standard example of an $E_{\infty}$ operad is $\mathscr{D}$, in which $\mathscr{D}_{S}$ is the nerve of the category $\mathbb{S} / S$ of isomorphisms of finite sets over $S$. Composition in $\mathscr{D}$ is induced by the deleted sum functor in $\mathbb{S}$.

\subsection{Cyclic operads}

A cyclic operad can be defined as an operad with extra structure (a $\Sigma_{n+1}$-action on $\left.\mathscr{C}_{\{1,2, \ldots, n\}}\right)$ which makes composition symmetric by putting the 'output' variable 0 on the same footing as the $n$ 'inputs' (see [7]). Then it is clearly desirable to change the notation, and denote by $\mathscr{C}_{S \sqcup 0}$ what was previously denoted $\mathscr{C}_{S}$. Confusion can arise, so we stress that from now on we shall use the 'cyclic' convention, and include the output in the labelling set, which now therefore has at least three elements.

It seems best to define cyclic operads directly. A cyclic operad is a functor $\mathscr{E}$ from the category of finite sets (with three or more elements) and isomorphisms, to the category of chain complexes; it also has composition operations

$$
\circ_{s, t}: \mathscr{E}_{S} \otimes \mathscr{E}_{T} \longrightarrow \mathscr{E}_{S \sqcup_{s, t} T}
$$

for all $S$ and $T$ and all choices of $s \in S, t \in T$. Here $S \sqcup_{s, t} T$ denotes the deleted sum $(S \backslash\{s\}) \sqcup(T \backslash\{t\})$ : we think of $s$ as the 'output' being fed into the 'input' labelled $t$. The composition $\circ_{s, t}$ is required to be a natural transformation of functors having the associativity property

$$
\circ_{s, t}\left(1 \otimes \circ_{t^{\prime}, u}\right)=\circ_{t^{\prime}, u}\left(\circ_{s, t} \otimes 1\right)
$$


for $s \in S, t, t^{\prime} \in T, t \neq t^{\prime}, u \in U$, and the symmetry property

$$
\tau_{*} \cdot \circ_{s, t}=\circ_{t, s} \cdot \tau_{\otimes}
$$

where $\tau_{*}: \mathscr{E}_{S \sqcup_{s, t} T} \approx \mathscr{E}_{T \sqcup_{t, s} S}$ is induced by the isomorphism of sets and $\tau_{\otimes}: \mathscr{E}_{S} \otimes \mathscr{E}_{T} \approx$ $\mathscr{E}_{T} \otimes \mathscr{E}_{S}$ is the signed interchange of factors

$$
\tau_{\otimes}(x \otimes y)=(-1)^{|x||y|}(y \otimes x) .
$$

Some further notation will be needed. The composition

$$
\circ_{s, t}: \mathscr{E}_{S} \otimes \mathscr{E}_{T} \longrightarrow \mathscr{E}_{V}
$$

is associated with a partition of $V$ into two subsets $S \backslash\{s\}$ and $T \backslash\{t\}$. Conversely, let $V=P \sqcup Q$ be any partition of $V$ into two sets, each with at least two elements. We can define the associated composition by writing $P^{1}$ and $Q^{2}$ for the disjoint unions $P \sqcup\{1\}$ and $Q \sqcup\{2\}$, and taking

$$
\circ_{12}: \mathscr{E}_{P^{1}} \otimes \mathscr{E}_{Q^{2}} \longrightarrow \mathscr{E}_{V}
$$

1·3. $E_{\infty}$ cyclic operads

We call $\mathscr{E}$ an $E_{\infty}$ cyclic operad if for all $S$ the complex $\mathscr{E}_{S}$ is contractible and $\Sigma_{S}$-free. It suffices to check this for $S=\{0,1, \ldots, n\}$ for all $n \geqslant 2$. We note that the $E_{\infty}$ condition is stronger than in $(1 \cdot 1)$, because the action of a larger symmetric group must be free. The operad $\mathscr{D}$ defined as in $(1 \cdot 1)$ is an $E_{\infty}$ cyclic operad, the composition again being induced by the deleted sum functor.

\subsection{Cofibrant operads}

We adopt the notation introduced in $(1 \cdot 2)$ for adding new points to a set: $S^{1}, S^{2}$ and $S^{12}$ are to denote $S \sqcup\{1\}, S \sqcup\{2\}$ and $S \sqcup\{1,2\}$, respectively. For each partition $V=S \sqcup T$ of $V$ we have a composition map $\circ_{21}: \mathscr{E}_{S^{2}} \otimes \mathscr{E}_{T^{1}} \rightarrow \mathscr{E}_{V}$. In a cofibrant operad, provided $S$ and $T$ each have more than one element, we want this map to be the inclusion of a face of $\mathscr{E}_{V}$, so we require it to be an (equivariant) cofibration; and we require faces to intersect only in faces of faces. This leads to the following.

Definition. Let $\partial \mathscr{E}_{V}$ denote the coequalizer of the maps

$$
\bigoplus_{V=P \sqcup Q \sqcup R} \mathscr{E}_{P^{2}} \otimes \mathscr{E}_{Q^{12}} \otimes \mathscr{E}_{R^{1}} \stackrel{\circ_{21} \otimes 1}{\underset{1 \otimes \circ_{21}}{\longrightarrow}} \bigoplus_{V=S \sqcup T} \mathscr{E}_{S^{2}} \otimes \mathscr{E}_{T^{1}},
$$

where the sums are indexed by partitions of $V$ into subsets of which $S$ and $T$, and hence $P$ and $R$, have at least two elements each. Associativity of composition implies that $\circ_{21}$ induces a $\Sigma_{V}$-equivariant map $\partial \mathscr{E}_{V} \rightarrow \mathscr{E}_{V}$, which we call the inclusion of the boundary. The cyclic operad $\mathscr{E}$ is cofibrant if for every $V$ the inclusion of the boundary is a $\Sigma_{V}$-equivariant cofibration. (The terminology is justified because these operads have the left lifting property with respect to those morphisms of operads which are surjective weak equivalences at every $S$. They are thus cofibrant in the sense of [14] and are additionally required to be cyclic.)

Cofibrant non-cyclic operads are defined in a completely analogous way. 


\subsection{The $E_{\infty}$ tree operad $\mathscr{T}$}

We now construct a cofibrant $E_{\infty}$ cyclic operad. The cyclic operad $\mathscr{D}$ will not do:

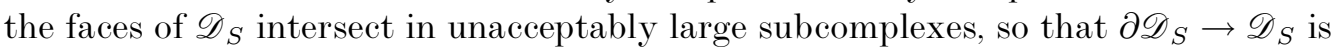
not injective. On the other hand, we can form another cyclic operad by taking $\mathscr{E}_{S}$ to be the space of trees with ends labelled by the set $S$, and $\circ_{s, t}$ to be the operation of grafting the end labelled $s$ to the end labelled $t$ to produce a new edge of length 1 . This operad has every $\mathscr{E}_{S}$ contractible [20], and it is cofibrant as the boundary is a subcomplex; but it is not an $E_{\infty}$ operad because $\Sigma_{n}$ does not act freely on $\mathscr{E}_{n}$.

By combining the two constructions we obtain a cofibrant $E_{\infty}$ cyclic operad, the tree operad $\mathscr{T}$, as follows. We take $\mathscr{T}_{S}$ to be the chain (bi)complex associated with the bisimplicial set in which a $(k, l)$-bisimplex consists of a $k$-simplex of the nerve of the category $\mathbb{S} / S$

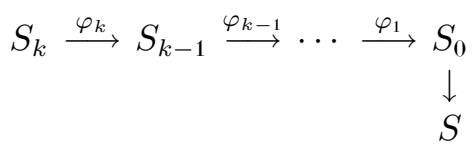

together with an $l$-simplex of the space $\tilde{T}_{S_{k}}$ of trees labelled by the set $S_{k}$; and the simplicial operators are defined in the obvious way. The composition maps $\circ_{s, t}$ in $\mathscr{T}$ are defined by using the deleted sum functor in the category $\mathbb{S}$ and the grafting of trees, as above.

Proposition 1·6. The operad $\mathscr{T}$ is cyclic, cofibrant and $E_{\infty}$.

Proof. It is clear that $\mathscr{T}$ is cyclic. It inherits the $E_{\infty}$ property of $\mathscr{D}$, because $\mathscr{T}_{S}$ is essentially the Borel construction $E \Sigma_{S} \times_{\Sigma_{S}} \mathscr{E}_{S}$ on the contractible space $\mathscr{E}_{S}$ of trees. We must show that $\mathscr{T}$ inherits the cofibrancy of the operad $\mathscr{E}$. In order to show that the inclusion of the boundary $\partial \mathscr{T}_{V}$ is an equivariant cofibration, it suffices to verify that it is injective and that $\Sigma_{V}$ acts freely on its complement. The freeness is clear, as $\Sigma_{V}$ acts freely on $\mathscr{T}_{V}$. For injectivity, let us simply note that a simplex lies in the face corresponding to a decomposition $V=S \sqcup T$ if and only if it consists of trees in which there is an internal edge, of maximal length, which separates the labels $S$ from the labels $T$; and therefore two faces meet only where two specified edges have maximal length, which is a face of a face (or is empty, as appropriate).

\section{Algebras, modules and realization}

\subsection{Algebras and modules over an operad}

Let $\mathscr{C}$ be a cyclic operad, and $K$ the ground ring, which is commutative with unit element.

Definition. An algebra over $\mathscr{C}$ is a chain complex (of $K$-modules) $A$ together with structural maps $\mu_{S}: \mathscr{C}_{S^{0}} \otimes A^{\otimes S} \rightarrow A$ for all sets $S$ (having two or more elements) which are natural in $S$ and satisfy the usual condition

$$
\mu_{S^{1}}\left(1 \otimes \mu_{T}\right)=\mu_{S \sqcup T}\left(\circ_{10} \otimes 1\right)
$$

of equality of maps $\mathscr{C}_{S^{01}} \otimes \mathscr{C}_{T^{0}} \otimes A^{\otimes(S \sqcup T)} \rightarrow A$.

By way of explanation we note that 0 has been adjoined to $S$ and $T$ as the "output variable' for the operad. The element 1 in $S^{01}$ is a dummy label associated with the partition $S \sqcup T$, as introduced at the end of $(1 \cdot 2)$. 
When the smallest model is chosen for the indexing category, which is the disjoint union of the symmetric groups $\Sigma_{n}$ for $n \geqslant 2$, the naturality condition in the definition simply means that $\mu_{n}$ is equivariant and so defines a map $\mathscr{C}_{n+1} \otimes_{\Sigma_{n}} A^{\otimes n} \rightarrow A$, where $\Sigma_{n}$ acts on $\mathscr{C}_{n+1}$ on the left (fixing the output label) and on $A^{\otimes n}$ on the right.

Definition. An $A$-module over $\mathscr{C}$, when $A$ is a $\mathscr{C}$-algebra as above, is a chain complex $M$ together with structural maps $\nu_{S}: \mathscr{C}_{S^{01}} \otimes A^{\otimes S} \otimes M \rightarrow M$ which are natural in $S$ and satisfy the usual module conditions

$$
\nu_{S}\left(1 \otimes \nu_{T}\right)=\nu_{S \sqcup T}\left(\circ_{10} \otimes 1\right)
$$

as maps $\mathscr{C}_{S^{01}} \otimes \mathscr{C}_{T^{01}} \otimes A^{\otimes(S \sqcup T)} \otimes M \rightarrow M$, and

$$
\nu_{S^{2}}\left(1 \otimes \mu_{T}\right)=\nu_{S \sqcup T}\left(\circ_{20} \otimes 1\right)
$$

as maps $\mathscr{C}_{S^{012}} \otimes \mathscr{C}_{T^{0}} \otimes A^{\otimes(S \sqcup T)} \otimes M \rightarrow M$.

The above algebras and modules are non-unital.

\subsection{Algebras and modules over an $E_{\infty}$ operad}

From now on it is a standing assumption (except where the reverse is stated) that all operads are cyclic and $E_{\infty}$. An algebra $A$ over such an operad $\mathscr{C}$ will be called an $E_{\infty}$ algebra. Since $\mathscr{C}$ is automatically augmented over the standard commutative algebra operad, the ground ring $K$ is an $E_{\infty}$ algebra.

For the purposes of this paper, it suffices to define subalgebras and submodules in a naive way as chain subcomplexes which are closed under the appropriate operad action. If $A$ is a subalgebra of $B$ over $\mathscr{C}$, there is an inclusion homomorphism $A \rightarrow B$, and we call $B$ an $A$-algebra over $\mathscr{C}$. We shall usually work with $K$-algebras, where $K$ is the ground ring regarded as an algebra over $\mathscr{C}$. The unit element of $K$ then serves as a unit for the algebra. When considering modules over a $K$-algebra $A$, we require the induced $K$-module structure to be the standard, strict one.

\subsection{Cyclic and non-cyclic complexes over an operad}

We now aim to construct the homotopical cotangent complex $\mathscr{K}(B / A ; M)$ when $B$ is an $E_{\infty} K$-algebra, $A$ a $K$-subalgebra and $M$ a $B$-module. (Although very different in appearance and in construction, this will play in our theory the role analogous to that played in André-Quillen theory by the cotangent complex of $[\mathbf{1}, \mathbf{1 8}]$.)

Our cotangent complex will be a filtered object obtained by glueing together the objects $\mathscr{C}_{V^{0}} \otimes B^{\otimes V} \otimes M$, where $V$ runs through the category $\mathbb{S}_{+}$. (There is also a cyclic version.) Conceptually it resembles the realization of a simplicial object, or the analogue described in [21]. Because the realization sometimes has to be applied to species other than the standard $B^{\otimes V} \otimes M$ (as in Section 7 below), it is worthwhile to formulate a definition of a more general object which can be realized. There are two separate cases to be considered, corresponding to $\Gamma$-homology and cyclic $\Gamma$-homology.

Definition $2 \cdot 4$. Let $\mathscr{C}$ be a cyclic operad. A cyclic $\mathscr{C}$-complex is a cofunctor $\mathscr{M}$ from the category $\mathbb{S}_{+}$to the category of chain complexes, together with the following further data which specify an action of $\mathscr{C}$ on $\mathscr{M}$ : for each composition

$$
\circ_{s, t}: \mathscr{C}_{S} \otimes \mathscr{C}_{T} \longrightarrow \mathscr{C}_{S \sqcup_{s, t} T}
$$


in $\mathscr{C}$, and also in the case where $T$ has only two elements, there is given a formal adjoint

$$
\circ_{s, t}^{*}: \mathscr{C}_{S} \otimes \mathscr{M}_{S \sqcup_{s, t} T} \longrightarrow \mathscr{M}_{T}
$$

(to be thought of as a cap product corresponding to the above cup product) which satisfies:

(1) the naturality condition

$$
\psi^{*} \circ_{s^{\prime}, t^{\prime}}^{*}\left(\varphi_{*} \otimes 1\right)=\circ_{s, t}^{*}\left(1 \otimes\left(\varphi \sqcup_{s, t} \psi\right)^{*}\right)
$$

for all isomorphisms $\varphi:(S, s) \approx\left(S^{\prime}, s^{\prime}\right), \psi:(T, t) \approx\left(T^{\prime}, t^{\prime}\right)$ in $\mathbb{S}$;

(2) the associativity condition

$$
\circ_{34}^{*}\left(1 \otimes \circ_{12}^{*}\right)=\circ_{34}^{*}\left(\circ_{21} \otimes 1\right): \mathscr{C}_{S^{32}} \otimes \mathscr{C}_{R^{1}} \otimes \mathscr{M}_{R \sqcup S \sqcup T} \longrightarrow \mathscr{M}_{T^{4}}
$$

for all finite sets $R^{1}, S^{23}, T^{4}$;

(3) the associativity condition

$$
\circ_{43}^{*}\left(1 \otimes \circ_{12}^{*}\right)=\circ_{12}^{*}\left(1 \otimes \circ_{43}^{*}\right)(\tau \otimes 1): \mathscr{C}_{T^{4}} \otimes \mathscr{C}_{R^{1}} \otimes \mathscr{M}_{R \sqcup S \sqcup T} \longrightarrow \mathscr{M}_{S^{23}}
$$

for all finite sets $R^{1}, S^{23}, T^{4}$, where $\tau$ interchanges factors.

There are two associativity conditions above for the same reason as in the definition of non-cyclic operad: there are two types of iterated substitution to be considered.

Example 2.5. Suppose $A$ is an algebra over the operad $\mathscr{C}$, with structural maps $\mu_{V}: \mathscr{C}_{V^{0}} \otimes A^{\otimes V} \rightarrow A$. Then we can take $\mathscr{M}_{S}=A^{\otimes S}$, and define $\circ_{s, t}^{*}$ to be

$$
\mathscr{C}_{S} \otimes A^{\otimes\left(S \sqcup_{s, t} T\right)} \approx \mathscr{C}_{S} \otimes A^{\otimes(S \backslash\{s\})} \otimes A^{\otimes(T \backslash\{t\})} \stackrel{\mu_{S \backslash\{s\}} \otimes 1}{\longrightarrow} A \otimes A^{\otimes(T \backslash\{t\})} \approx A^{\otimes T}
$$

giving a cyclic $\mathscr{C}$-complex.

Now we need the non-cyclic version, which is slightly more complicated because the indexing sets have basepoints and there are, as in the definition of operads, correspondingly more cases to consider. (The basepoint may be in any subset of a partition.) We consistently write 0 for the basepoint, so that a typical based finite set is $S^{0}$, where $S$ is an object of $\mathbb{S}$.

Definition 2.6. Let $\mathscr{C}$ be a cyclic operad. A (non-cyclic) $\mathscr{C}$-complex assigns to each based finite set $S^{0}$ a chain complex $\mathscr{M}_{S^{0}}$ depending cofunctorially upon $S^{0}$, and to each composition

$$
\circ_{0,1}: \mathscr{C}_{S^{0}} \otimes \mathscr{C}_{T^{01}} \longrightarrow \mathscr{C}_{(S \sqcup T)^{0}}
$$

in $\mathscr{C}$ a pair of formal adjoints

$$
\circ_{0,1}^{*}: \mathscr{C}_{S^{0}} \otimes \mathscr{M}_{(S \sqcup T)^{0}} \longrightarrow \mathscr{M}_{T^{01}}
$$

and

$$
\circ_{1,0}^{*}: \mathscr{C}_{T^{01}} \otimes \mathscr{M}_{(S \sqcup T)^{0}} \longrightarrow \mathscr{M}_{S^{0}}
$$

(existing even when $T^{01}$ or $S^{0}$, respectively has only two elements) satisfying the analogues of the naturality and associativity conditions (1)-(3) of Definition 2.4. The asymmetry in this definition arises because the composition $\circ_{0,1}$ corresponds to a partition of $V^{0}=(S \sqcup T)^{0}=S \sqcup T^{0}$ into a subset which does not contain the basepoint and one which does. The first of the two adjoints above evaluates over $S$, 
and takes values in $\mathscr{M}_{T^{01}}$, where 1 is a dummy label from the partition; the second evaluates over $T^{0}$, and takes values in $\mathscr{M}_{S^{0}}$, where 0 is a new dummy basepoint for $S$.

Example $2 \cdot 7$. Just as the primary example of a cyclic $\mathscr{C}$-complex arises from an algebra (Example $2 \cdot 5$ ), so the primary example of a non-cyclic $\mathscr{C}$-complex arises from a module. In Definition $2 \cdot 6$ above, let $\mathscr{M}_{S^{0}}=A^{\otimes S} \otimes M$ where $A$ is a $K$-algebra over $\mathscr{C}$ and $M$ an $A$-module; let $\circ_{0,1}^{*}$ be $\mu_{S} \otimes 1$, where $\mu$ is the algebra structure, and $\circ_{1,0}^{*}$ be $1 \otimes \nu_{T}$, where $\nu$ is the module structure.

\subsection{The realization of a $\mathscr{C}$-complex in the non-cyclic case}

Let $\mathscr{C}$ be a cofibrant cyclic operad, and $\mathscr{M}$ a (non-cyclic) $\mathscr{C}$-complex. We construct the realization $|\mathscr{M}|$ by a process resembling that for realizing a simplicial set. We treat the non-cyclic case in detail, because it is more important for us, then describe the differences in the cyclic case, which is important in cyclic $\Gamma$-homology. There are two steps in the construction.

First we construct a complex $|\mathscr{M}|^{\prime}$. We take a direct sum over all $V^{0}=V \sqcup\{0\}$ in $\mathbb{S}^{1}$ (our category of based sets) having three or more elements

$$
\bigoplus_{\left|V^{0}\right| \geqslant 3} \mathscr{C}_{V^{0}} \otimes \mathscr{M}_{V^{0}}
$$

$|\mathscr{M}|^{\prime}$ is the quotient of this by the following identifications:

(1) for each isomorphism $\varphi: S^{0} \approx T^{0}$ in $\mathbb{S}^{1}$, and all $x \in \mathscr{C}_{S^{0}}$, all $m \in \mathscr{M}_{T^{0}}$

$$
\varphi_{*} x \otimes m \sim x \otimes \varphi^{*} m
$$

(2) for each partition $V^{0}=S \sqcup T^{0}$ of a set into two subsets (the second containing the basepoint) having at least two elements each, we consider the associated composition (as in $(1 \cdot 2)$ )

$$
\circ_{0,1}: \mathscr{C}_{S^{0}} \otimes \mathscr{C}_{T^{01}} \longrightarrow \mathscr{C}_{(S \sqcup T)^{0}}=\mathscr{C}_{V^{0}}
$$

and we define

$$
\partial^{S, T}: \mathscr{C}_{S^{0}} \otimes \mathscr{C}_{T^{01}} \otimes \mathscr{M}_{(S \sqcup T)^{0}} \longrightarrow \mathscr{C}_{S^{0}} \otimes \mathscr{M}_{S^{0}} \oplus \mathscr{C}_{T^{01}} \otimes \mathscr{M}_{T^{01}}
$$

by setting

$$
\partial^{S, T}=\left(1 \otimes \circ_{10}^{*}\right) \oplus\left(1 \otimes \circ_{01}^{*}\right)(\tau \otimes 1)
$$

where $\tau$ interchanges the factors $\mathscr{C}_{S^{0}}$ and $\mathscr{C}_{T^{01}}$ and introduces the usual sign. Then on the component of the boundary $\partial \mathscr{C}_{V^{0}} \otimes \mathscr{M}_{V^{0}}$ corresponding to $\circ_{0,1}$ we make identifications by requiring

$$
\circ_{01}(x \otimes y) \otimes m \sim \partial^{S, T}(x \otimes y \otimes m)
$$

for all $x \in \mathscr{C}_{S^{0}}, y \in \mathscr{C}_{T^{01}}, m \in \mathscr{M}_{V^{0}}$.

The complex $|\mathscr{M}|^{\prime}$ thus defined is a quotient of

$$
\bigoplus_{n \geqslant 2} \mathscr{C}_{n+1} \otimes_{\Sigma_{n}} \mathscr{M}_{n+1}
$$

where $n+1$ denotes the $(n+1)$-element based set $\{0,1, \ldots, n\}$. This is because the identifications (1) imply that it suffices to take one indexing set of each size, 
and pass to the quotient by the action of $\Sigma_{n}$. We can define the skeletal filtration of $|\mathscr{M}|^{\prime}$ by defining the $k$-skeleton to be the image of $\bigoplus_{2 \leqslant n \leqslant k} \mathscr{C}_{n+1} \otimes \mathscr{M}_{n+1}$. Just as in the standard simplicial realization construction, the identifications (2) satisfy compatibility conditions which guarantee that the $k$ th filtration quotient of $|\mathscr{M}|^{\prime}$ is isomorphic to $\left(\mathscr{C}_{k+1} / \partial \mathscr{C}_{k+1}\right) \otimes_{\Sigma_{k}} \mathscr{M}_{k+1}$.

We now describe the second step in the construction, which incorporates the bottom filtration stage $\mathscr{M}_{2}$. (If $\mathscr{C}_{2}$ were defined, it would be contractible for an $E_{\infty}$ operad. Thus $\mathscr{M}_{2}$ would be quasi-isomorphic to $\mathscr{C}_{2} \otimes_{\Sigma_{1}} \mathscr{M}_{2}$.)

Let $V^{0}$ be any based set in $\mathbb{S}^{1}$ having three or more elements. Take any $v \in V$, and write $T_{v}$ for $V \backslash\{v\}$. The partition $V^{0}=\{v\} \sqcup T_{v}$ has an associated composition

$$
\circ_{0,1}: \mathscr{C}_{\{v, 0\}} \otimes \mathscr{C}_{T_{v}^{01}} \longrightarrow \mathscr{C}_{V^{0}}
$$

and action

$$
\circ_{1,0}^{*}: \mathscr{C}_{T_{v}^{01}} \otimes \mathscr{M}_{V^{0}} \longrightarrow \mathscr{M}_{\{v, 0\}} .
$$

The standard isomorphisms $T_{v}^{01}=V \backslash\{v\} \sqcup\{0,1\} \approx V^{0}$ and $\{v, 0\} \approx\{0,1\}$, taking $v$ to 1 in each case, convert this action into a map

$$
\varepsilon_{v}: \mathscr{C}_{V^{0}} \otimes \mathscr{M}_{V^{0}} \longrightarrow \mathscr{M}_{\{0,1\}}=\mathscr{M}_{2} .
$$

If instead of $v \in V$ we select $0 \in V^{0}$, the other action map $\circ_{0,1}^{*}$ (defined in Definition $2 \cdot 6)$ yields in identical fashion a map

$$
\varepsilon_{0}: \mathscr{C}_{V^{0}} \otimes \mathscr{M}_{V^{0}} \longrightarrow \mathscr{M}_{\{0,1\}}=\mathscr{M}_{2} .
$$

Let $\varepsilon=\varepsilon_{0}-\sum_{v \in V} \varepsilon_{v}$. This defines, for each $V^{0}$ in $\mathbb{S}^{1}$ having three or more elements, a map $\mathscr{C}_{V^{0}} \otimes \mathscr{M}_{V^{0}} \rightarrow \mathscr{M}_{2}$. One can check immediately that the naturality and associativity conditions in the definition of a $\mathscr{C}$-complex imply that these maps are compatible with the identifications used in the construction of $|\mathscr{M}|^{\prime}$. Therefore we have a well-defined map $\varepsilon:|\mathscr{M}|^{\prime} \rightarrow \mathscr{M}_{2}$. The final realization $|\mathscr{M}|$ is defined to be the cofibre of $\varepsilon$. This completes the construction of the realization in the non-cyclic case.

\subsection{Realization in the cyclic case}

Now let $\mathscr{M}$ be a cyclic $\mathscr{C}$-complex, where $\mathscr{C}$ is a cofibrant cyclic operad. We construct the cyclic realization $|\mathscr{M}|_{\text {cy }}$ by modifying the construction of $(2 \cdot 8)$ as follows. We begin with a sum indexed by all $V$ in our category $\mathbb{S}$ of unbased finite sets containing at least three elements

$$
\bigoplus_{|V| \geqslant 3} \mathscr{C}_{V} \otimes \mathscr{M}_{V}
$$

We alter the identifications to take account of the extra symmetry available in that there is now no basepoint: they now read

(1) for each isomorphism $\varphi: S \approx T$ in $\mathbb{S}$, and all $x \in \mathscr{C}_{S}$, all $m \in \mathscr{M}_{T}$

$$
\varphi_{*} x \otimes m \sim x \otimes \varphi^{*} m .
$$

(2) for each partition $V=S \sqcup T$ of a set into two subsets having at least two elements each, we define

$$
\partial^{S, T}: \mathscr{C}_{S^{2}} \otimes \mathscr{C}_{T^{1}} \otimes \mathscr{M}_{(S \sqcup T)} \longrightarrow \mathscr{C}_{S^{2}} \otimes \mathscr{M}_{S^{2}} \oplus \mathscr{C}_{T^{1}} \otimes \mathscr{M}_{T^{1}}
$$


by setting

$$
\partial^{S, T}=\left(1 \otimes \circ_{12}^{*}\right) \oplus\left(1 \otimes \circ_{21}^{*}\right)(\tau \otimes 1)
$$

where $\tau$ interchanges the factors $\mathscr{C}_{S^{2}}$ and $\mathscr{C}_{T^{1}}$ and introduces the usual sign. Then on the component of the boundary $\partial \mathscr{C}_{V} \otimes \mathscr{M}_{V}$ corresponding to $\circ_{2,1}$ we make identifications by requiring

$$
\circ_{21}(x \otimes y) \otimes m \sim \partial^{S, T}(x \otimes y \otimes m)
$$

for all $x \in \mathscr{C}_{S^{2}}, y \in \mathscr{C}_{T^{1}}, m \in \mathscr{M}_{V}$.

We have now completed the description of the first stage, which we denote $|\mathscr{M}|_{\text {cy }}^{\prime}$, of the cyclic realization.

The identifications above mean in effect that $|\mathscr{M}|^{\prime}$ is a quotient of

$$
\bigoplus_{n \geqslant 2} \mathscr{C}_{n+1} \otimes_{\Sigma_{n+1}} \mathscr{M}_{n+1}
$$

where $\Sigma_{n+1}$ is the group of permutations of $\{0,1, \ldots, n\}$.

In analogy with $(2 \cdot 8)$ we now expect to define a map

$$
\varepsilon:|\mathscr{M}|_{\text {cy }}^{\prime} \longrightarrow \mathscr{C}_{2} \otimes_{\Sigma_{2}} \mathscr{M}_{2}
$$

the cofibre of which would be $|\mathscr{M}|_{\text {cy }}$. We now proceed to explain that a sign intervenes in the representation, and that we shall replace the undefined $\mathscr{C}_{2}$ by a different contractible free $\Sigma_{2}$-complex.

The nerve of the category of isomorphisms of two-element sets is a model for the classifying space $B \Sigma_{2}$, and the nerve of the category of isomorphisms of ordered two-element sets is its universal cover $E \Sigma_{2}$. Let $V$ be any set in $\mathbb{S}$ having three or more elements. Take any $v \in V$, and write $T_{v}$ for $V \backslash\{v\}$. As in (2.8) we have a composition

$$
\circ_{0,1}: \mathscr{C}_{\{v, 0\}} \otimes \mathscr{C}_{T_{v}^{1}} \longrightarrow \mathscr{C}_{V}
$$

and action

$$
\mathrm{o}_{1,0}^{*}: \mathscr{C}_{T_{v}^{1}} \otimes \mathscr{M}_{V} \longrightarrow \mathscr{M}_{\{v, 0\}} .
$$

Using the isomorphism $T_{v}^{1} \approx V$, we obtain from the adjoint $\circ_{1,0}^{*}$ a map which we shall denote

$$
\circ_{v, 0}^{*}: \mathscr{C}_{V} \otimes \mathscr{M}_{V} \longrightarrow \mathscr{M}_{\{v, 0\}} .
$$

As $\{0, v\}$ is an ordered two-element set, we can regard it as a chain of $E \Sigma_{2}$. We define

$$
\tilde{\varepsilon}: \mathscr{C}_{V} \otimes \mathscr{M}_{V} \longrightarrow E \Sigma_{2} \otimes \mathscr{M}_{\{v, 0\}}
$$

by setting

$$
\tilde{\varepsilon}(x)=\sum_{v \in V}\left(\{0, v\} \otimes \circ_{v, 0}^{*}(x)\right) .
$$

This does not yet respect the identifications defining $|\mathscr{M}|_{\text {cy. }}^{\prime}$. But if we denote by $\tilde{\mathscr{M}}_{2}$ the complex $\mathscr{M}_{2}$ with its $\Sigma_{2}$-structure twisted by the sign representation, then $\tilde{\varepsilon}$ composes with the quotient map to give a well-defined map

$$
\varepsilon:|\mathscr{M}|_{\text {cy }}^{\prime} \longrightarrow E \Sigma_{2} \otimes_{\Sigma_{2}} \tilde{\mathscr{M}}_{2} .
$$

We finally define the cyclic realization $|\mathscr{M}|_{\text {cy }}$ to be the cofibre of this map. 
Remarks $2 \cdot 10$. (1) The sign in the last stage of the above construction is needed to ensure cancellation of the unwanted contributions from the two dummy labels in a partition as in identification $(2 \cdot 9(2))$ above.

(2) There is a natural map $|\mathscr{M}| \rightarrow|\mathscr{M}|_{\text {cy }}$ induced by the levelwise quotient maps $\mathscr{C}_{n+1} \otimes_{\Sigma_{n}} \mathscr{M}_{n+1} \rightarrow \mathscr{C}_{n+1} \otimes_{\Sigma_{n+1}} \mathscr{M}_{n+1}$, which are well-behaved with respect to the identifications in the construction.

2.11. Uniqueness of $E_{\infty}$ realization

We now prove that the homotopy type of the realization $|\mathscr{M}|$ or $|\mathscr{M}|_{\text {cy }}$ does not depend upon the cofibrant cyclic operad $\mathscr{C}$ used to construct it, provided that $\mathscr{C}$ is $E_{\infty}$. The proof uses the standard idea of comparison of resolutions.

Lemma. Let $\mathscr{C}$ and $\mathscr{D}$ be $E_{\infty}$ cyclic operads, with $\mathscr{C}$ cofibrant. Then there is a map $\mathscr{C} \rightarrow \mathscr{D}$ of cyclic operads, and it is unique up to homotopy.

Proof. We construct $\Sigma_{n+1}$-equivariant maps $\varphi_{n+1}: \mathscr{C}_{n+1} \rightarrow \mathscr{D}_{n+1}$, commuting with all composition maps, by using induction on $n$.

There are no decomposable elements in $\mathscr{C}_{3}$, so we start by taking $\varphi_{3}: \mathscr{C}_{3} \rightarrow \mathscr{D}_{3}$ to be any $\Sigma_{3}$-equivariant map. This map exists because $\mathscr{C}_{3}$ is free and $\mathscr{D}_{3}$ is contractible. Suppose by inductive hypothesis that we have equivariant $\varphi_{k+1}$ for all $k<n$, commuting with compositions as far as this makes sense. We have to define $\varphi_{n+1}$. The boundary $\partial \mathscr{C}_{n+1}$ is by (1.4) a sum of copies of $\mathscr{C}_{i+1} \otimes \mathscr{C}_{j+1}$ with $2 \leqslant i, j$ and $i+j=n+1$, amalgamated along $\mathscr{C}_{i+1} \otimes \partial \mathscr{C}_{j+1} \cup \partial \mathscr{C}_{i+1} \otimes \mathscr{C}_{j+1}$. The maps $\varphi_{i+1} \otimes \varphi_{j+1}$ therefore induce a map $\partial \mathscr{C}_{n+1} \rightarrow \mathscr{D}_{n+1}$, equivariant with respect to the induced action of $\Sigma_{n+1}$. Since $\mathscr{C}$ is cofibrant and $\mathscr{D}_{n+1}$ is contractible, this map extends to a $\Sigma_{n+1}$-equivariant map $\mathscr{C}_{n+1} \rightarrow \mathscr{D}_{n+1}$, which by construction retains the compatibility with compositions. The inductive proof of existence is now complete. The proof of homotopy uniqueness is similar.

Proposition 2.12. If $\mathscr{M}$ is a complex over one $E_{\infty}$ cyclic operad, then it is a complex over every cofibrant cyclic $E_{\infty}$ operad, and the homotopy type of the realization $|\mathscr{M}|$ (or $|\mathscr{M}|_{\text {cy }}$, in the cyclic case) is independent of the cyclic cofibrant $E_{\infty}$ operad used to construct it.

Proof. Let $\mathscr{C}$ and $\mathscr{D}$ be cyclic $E_{\infty}$ operads, with $\mathscr{C}$ cofibrant. By $(2 \cdot 11)$ there is a map of operads, unique up to homotopy, from $\mathscr{C}$ to $\mathscr{D}$. If $\mathscr{M}$ is a $\mathscr{D}$-complex, such a map induces the structure (unique up to homotopy) of a $\mathscr{C}$-complex on $\mathscr{M}$.

Suppose now that $\varphi: \mathscr{C} \rightarrow \mathscr{D}$ is a map of $E_{\infty}$ operads. We show by induction on $k$ that $\varphi_{k+1}$ is a homotopy equivalence of pairs $\left(\mathscr{C}_{k+1}, \partial \mathscr{C}_{k+1}\right) \rightarrow\left(\mathscr{D}_{k+1}, \partial \mathscr{D}_{k+1}\right)$. This is certainly true for $k=1,2$, where the spaces are contractible and the boundaries are empty. Suppose it is true for $k<n$. The assembly of $\partial \mathscr{C}_{n+1}$ and $\partial \mathscr{D}_{n+1}$ from cofibrations of lower spaces in the operads (as in the proof of $(2 \cdot 11)$ ) implies that $\varphi_{n+1}$ restricts to a homotopy equivalence $\partial \mathscr{C}_{n+1} \rightarrow \partial \mathscr{D}_{n+1}$. But then $\mathscr{C}_{n+1}$ and $\mathscr{D}_{n+1}$ are contractible, and the inclusions of the boundaries are cofibrations, so $\varphi_{n+1}$ is a homotopy equivalence of pairs.

When $\mathscr{M}$ has the $\mathscr{C}$-structure induced by the map $\varphi$, there is a skeleton-preserving induced map $|\varphi|:|\mathscr{M}|_{\mathscr{C}} \rightarrow|\mathscr{M}|_{\mathscr{D}}$ between the realizations constructed using the two different operads. On quotients of adjacent skeleta, $|\varphi|$ induces a map

$$
\left(\mathscr{C}_{n} / \partial \mathscr{C}_{n}\right) \otimes_{\Sigma_{n}} \mathscr{M}_{n} \stackrel{\varphi_{n} \otimes 1}{\longrightarrow}\left(\mathscr{D}_{n} / \partial \mathscr{D}_{n}\right) \otimes_{\Sigma_{n}} \mathscr{M}_{n}
$$


which is a homotopy equivalence because $\varphi_{n}$ has been shown to be a homotopy equivalence of free $\Sigma_{n}$-complexes. By induction and direct limit, $|\varphi|$ is a homotopy equivalence. Hence $|\mathscr{M}|$ is independent of the cofibrant $E_{\infty}$ operad used. A similar proof works in the cyclic case.

\subsection{The homology of the realization}

Proposition.

(1) Let $\mathscr{M}$ be a $\mathscr{C}$-complex, where $\mathscr{C}$ is an $E_{\infty}$ cyclic operad. Then there is a homology spectral sequence

$$
E_{p-1, q}^{1} \approx H_{q}\left(E \Sigma_{p} \otimes_{\Sigma_{p}}\left(V_{p} \otimes \mathscr{M}_{p+1}\right)\right) \Longrightarrow H_{p+q-1}(|\mathscr{M}|),
$$

where $V_{p}$ is the representation of $\Sigma_{p}$ on the homology of the tree space $T_{p}$, and $\Sigma_{p}$ acts diagonally on $V_{p} \otimes \mathscr{M}_{p+1}$.

(2) When $\mathscr{M}$ is a cyclic $\mathscr{C}$-complex there is a corresponding homology spectral sequence of the form

$$
E_{p-1, q}^{1} \approx H_{q}\left(E \Sigma_{p+1} \otimes_{\Sigma_{p+1}}\left(V_{p}^{\prime} \otimes \mathscr{M}_{p+1}\right)\right) \Longrightarrow H_{p+q-1}\left(|\mathscr{M}|_{c y}\right),
$$

where $V_{p}^{\prime}$ is the integral representation of $\Sigma_{p+1}$ on the homology of $T_{p}$.

Proof. We know from (2.8) that the $n$th filtration quotient in the skeletal filtration of $|\mathscr{M}|$ is $\left(\mathscr{C}_{n} / \partial \mathscr{C}_{n}\right) \otimes_{\Sigma_{n}} \mathscr{M}_{n}$. By Proposition $2 \cdot 12|\mathscr{M}|$ is independent, up to filtered quasi-isomorphism, of the choice of operad. Therefore the spectral sequence obtained from the skeletal filtration is independent, from the $E^{1}$ term onward, of the particular cofibrant cyclic $E_{\infty}$ operad used in the construction. Choosing the $E_{\infty}$ tree operad of $(1.5)$ leads formulae given above. The argument in the cyclic case is completely analogous.

It is worth noting here that the above $E_{p-1, *}^{1}$ term is in effect the hyperhomology of the group $\Sigma_{p}$ or $\Sigma_{p+1}$ with coefficients in the complex $V_{p} \otimes \mathscr{M}_{p+1}$ or $V_{p}^{\prime} \otimes \mathscr{M}_{p+1}$. Naturally we have the hyperhomology spectral sequence

$$
H_{s}\left(\Sigma_{p} ; V_{p} \otimes H_{t}\left(\mathscr{M}_{p+1}\right)\right) \Longrightarrow E_{p-1, s+t}^{1}
$$

and its analogue for the second case, available as subsidiary spectral sequences for calculating the $E^{1}$-terms.

It is known that the representation $V_{p}$ is isomorphic over $\mathbb{Z}$ to $\operatorname{Hom}\left(\operatorname{Lie}_{p}, \mathbb{Z}[-1]\right)$, where $\operatorname{Lie}_{p}$ is the Lie representation and $\mathbb{Z}[-1]$ the sign representation. The module $V_{p}^{\prime}$ is related to $V_{p}$ and therefore to the Lie representations by a short exact sequence

$$
0 \longrightarrow V_{p+1} \longrightarrow \operatorname{Ind}_{\Sigma_{p}}^{\Sigma_{p+1}} V_{p} \longrightarrow V_{p}^{\prime} \longrightarrow 0 .
$$

The complex character of $V_{p}^{\prime}$ is calculated in [20]; information about the integral representations may be found in [23].

\section{The $\Gamma$-cotangent complex and the transitivity theorem}

\subsection{Introduction}

We now apply the general theory of Section 2 to the case we are really interested in: the construction of the $\Gamma$-cotangent complex $\mathscr{K}(B / A ; M)$ when $A$ is a subalgebra of the $E_{\infty}$ differential graded algebra $B$, and $M$ is any $B$-module. In the construction 
we shall use any cofibrant $E_{\infty}$ cyclic operad $\mathscr{C}$, such as the tree operad $\mathscr{T}$ of $(1 \cdot 5)$ : the result is independent, up to quasi-isomorphism, of the choice. It is no real loss of generality to assume that $A, B$ and $M$ are flat or even projective over the ground ring $K$ : the structure of algebra or module over a cofibrant operad is homotopy invariant, so that one can replace these objects by projective resolutions; and our realizations are homotopy invariant constructions, so the choice of projective resolution makes no difference to the result. Similarly we may assume that $A \subset B$ is a cofibration.

We also prove a flat base-change result, showing that $\mathscr{K}(B / A ; M)$ is essentially independent of the ground ring.

The notation of the above introduction will be used throughout the section.

Let $\mathscr{K}$ be the following non-cyclic $\mathscr{C}$-complex, which was mentioned in Example $2 \cdot 7$. For each based finite set $V^{0}=V \sqcup\{0\}$ in $\mathbb{S}^{1}$ we put

$$
\mathscr{K}_{V^{0}}=A^{\otimes V} \otimes M .
$$

For every partition $V^{0}=S \sqcup T^{0}$ of $V^{0}$ into nonempty sets we define

$$
\circ_{0,1}^{*}: \mathscr{C}_{S^{0}} \otimes \mathscr{K}_{(S \sqcup T)^{0}} \longrightarrow \mathscr{K}_{T^{10}}
$$

by using the algebra structure map $\mu$

$$
\circ_{0,1}^{*}=\mu_{S} \otimes 1 \otimes 1: \mathscr{C}_{S^{0}} \otimes A^{\otimes S} \otimes A^{\otimes T} \otimes M \longrightarrow A \otimes A^{\otimes T} \otimes M,
$$

and we define

$$
\circ_{1,0}^{*}: \mathscr{C}_{T^{10}} \otimes \mathscr{K}_{(S \sqcup T)^{0}} \longrightarrow \mathscr{K}_{S^{0}}
$$

by using the module structure map $\nu$

$$
\circ_{1,0}^{*}=1 \otimes \nu_{T}: A^{\otimes S} \otimes \mathscr{C}_{T^{10}} \otimes A^{\otimes T} \otimes M \longrightarrow A^{\otimes S} \otimes M .
$$

It follows from the algebra and module axioms that this is a $\mathscr{C}$-complex. Its realization $|\mathscr{K}|$ we denote usually by $\mathscr{K}_{K}(A ; M)$, in order to stress that it depends in an essential way upon the ground ring $K$.

\section{3·2. $\Gamma$-cotangent complex and $\Gamma$-homology groups}

Let $A$ be a subalgebra of the $E_{\infty}$ algebra $B$, as in (3.1), and $M$ a $B$-module, all these being assumed flat over $K$. We define the $\Gamma$-cotangent complex of $B$ relative to $A$, with coefficients $M$ to be the quotient

$$
\mathscr{K}(B / A ; M)=\mathscr{K}_{K}(B ; M) / \mathscr{K}_{K}(A ; M) .
$$

(Here we have assumed that $A \subset B$ is a cofibration. In a more general situation the quotient must be replaced by the cofibre.) The $\Gamma$-homology of $B$ over $A$ is the homology of this complex:

$$
H \Gamma_{*}(B / A ; M)=H_{*}(\mathscr{K}(B / A ; M)) ;
$$

the $\Gamma$-cohomology is correspondingly defined by

$$
H \Gamma^{*}(B / A ; M)=H^{*}\left(囚 \operatorname{Hom}_{B}(\mathscr{K}(B / A ; B), M) .\right.
$$

Remark 3.3. For theoretical and practical reasons we have chosen to define the cotangent complex as a quotient. This avoids the need to handle derived tensor products over $E_{\infty}$ algebras. It also has the pleasant consequence that the important Transitivity Theorem $3 \cdot 4$ (below) becomes trivial to prove. This advantage is 
of course an illusion: the counterbalancing disadvantage is that in order for our definitions to be useful, we have to work to prove that $\mathscr{K}(B / A ; M)$, and hence $H \Gamma_{*}(B / A ; M)$, are essentially independent of the ground ring $K$. The proof of this flat base-change theorem occupies most of the rest of Section 3, and the Appendix to this paper.

Transitivity Theorem 3.4. Let $A \subset B \subset C$ be inclusions of $E_{\infty}$-algebras, and $M$ an $E_{\infty} C$-module. Then there is a cofibration sequence of $\Gamma$-cotangent complexes

$$
\mathscr{K}(B / A ; M) \longrightarrow \mathscr{K}(C / A ; M) \longrightarrow \mathscr{K}(C / B ; M) \longrightarrow S \mathscr{K}(B / A ; M)
$$

and therefore a long exact sequence of $\Gamma$-homology groups

$$
\begin{aligned}
\cdots \longrightarrow H \Gamma_{n+1}(C / B ; M) \longrightarrow H \Gamma_{n}(B / A ; M) & \longrightarrow H \Gamma_{n}(C / A ; M) \\
& \longrightarrow H \Gamma_{n}(C / B ; M) \longrightarrow \cdots
\end{aligned}
$$

and similarly for cohomology.

Proof. The definitions require $A, B$ and $C$ to be replaced by corresponding projective resolutions. Then everything follows from the exact sequence connecting the cofibres of the three maps in the triple

$$
\mathscr{K}_{K}(A ; M) \longrightarrow \mathscr{K}_{K}(B ; M) \longrightarrow \mathscr{K}_{K}(C ; M) .
$$

We now begin on the definitions and lemmas which we shall need for the other main result of this section, the flat base-change theorem.

\subsection{A model for the derived tensor product}

We propose that the derived tensor product of modules over an $E_{\infty}$ algebra should be defined by the following construction. For the case of modules over a commutative algebra, which is the only case we shall use in this paper, we reconcile the definition with a standard one in Proposition 3.6.

Let $\mathscr{C}$ be a cofibrant cyclic $E_{\infty}$ operad. We are going to make a realization like those in $(2 \cdot 8)$ and $(2 \cdot 9)$, but with $\mathbb{S}$ or $\mathbb{S}^{1}$ replaced by $\mathbb{S}^{r}$, which is the category of finite sets with $r$ distinct basepoints $0_{1}, \ldots, 0_{r}$, and isomorphisms of sets which preserve the basepoints in order. There is formally no problem in extending the definition given in (2.8) to this case, except that when $S \sqcup T$ is a partition of the set $V=\hat{V} \sqcup\left\{0_{1}, \ldots, 0_{r}\right\}$ in $\mathbb{S}^{r}$, the structural map

$$
\circ_{p q}: \mathscr{C}_{S^{p}} \otimes \mathscr{M}_{S \sqcup T} \longrightarrow \mathscr{M}_{T^{q}}
$$

must be zero when $S$ contains more than one of the basepoints, as there is then no natural way to structure $T^{q}$ as a space with $r$ basepoints. (Here $p$ and $q$ are dummy labels.) When $r \geqslant 2$, the homology of the realization is much simpler than the homologies for which we obtained spectral sequences in $(2 \cdot 13)$, because the analogues of $V_{p}$ are now free $\Sigma_{p}$-modules. That is why the following construction is valid.

Let $A$ be a $\mathscr{C}$-algebra and let $M_{1}, \ldots, M_{r}$ be $A$-modules. Suppose that $A$ and all the $M_{i}$ are flat over the commutative ground ring $K$. We construct a $\mathscr{C}$-complex $\mathscr{M}$ on the category $\mathbb{S}^{r}$ by setting $\mathscr{M}_{V}=M_{1} \otimes \cdots \otimes M_{r} \otimes A^{\otimes \hat{V}}$ when $V=\hat{V} \sqcup\left\{0_{1}, \ldots, 0_{r}\right\}$ is a set with $r$ basepoints, and, when $V=S \sqcup T$, taking

$$
\circ_{p q}: \mathscr{C}_{S^{p}} \otimes \mathscr{M}_{S \sqcup T} \longrightarrow \mathscr{M}_{T^{q}}
$$


to be

$$
\circ_{p q}= \begin{cases}1 \otimes \mu_{S}, & \text { if } S \text { contains no basepoint } \\ 1 \otimes \nu_{S \backslash 0_{i}}^{i} & \text { if } S \text { contains } 0_{i} \text { and no other } \\ 0, & \text { if } S \text { contains more than one basepoint, }\end{cases}
$$

where $\mu$ is the structural map for the $\mathscr{C}$-algebra $A$, and $\nu^{i}$ is that for the module $M_{i}$.

We define the left derived tensor product $M_{1} \stackrel{L}{\otimes}_{A} M_{2} \stackrel{L}{\otimes}_{A} \ldots \stackrel{L}{\otimes}_{A} M_{r}$ to be the realization $|\mathscr{M}|$.

The following proposition justifies the above definition in the case of tensor products over a commutative algebra, where we have a classical definition with which to compare ours.

Proposition 3.6. Let $R$ be a commutative algebra over the ground ring $K$, and let $M_{1}, \ldots, M_{r}$ (where $r \geqslant 2$ ) be complexes of $R$-modules, in the sense of standard homological algebra. Suppose that $R$, and all the $M_{i}$ with at most one exception, are flat over $K$. Then the complex $M_{1} \otimes_{R}^{L} M_{2} \otimes_{R}^{L} \cdots \stackrel{L}{\otimes}_{R} M_{r}$ defined in (3.5) is quasi-isomorphic to the usual derived tensor product over $R$ of $M_{1}, \ldots, M_{r}$.

Proof. For transparency we treat first the case when $r=2$. After Proposition $2 \cdot 12$, we may assume that $\mathscr{C}$ is the tree operad of $(1 \cdot 5)$. We shall also need the corresponding $A_{\infty}$ operad, in which the $\mathscr{C}_{\text {ord }, n+2}$ is the chain complex of trees which can be embedded in the plane with labels $0_{1}, 1,2, \ldots, n, 0_{2}$ in cyclic order. This operad has no permutations. It is well known that $\mathscr{C}_{\text {ord }}$ is a subdivision of the Stasheff operad of associahedra. Using instead the standard cellular chains of the Stasheff polyhedra we deduce that the realization $|\mathscr{M}|_{\text {ord }}$ of the complex

$$
\mathscr{M}_{0_{1}, 1,2, \ldots, n, 0_{2}}=M_{1} \otimes R^{\otimes n} \otimes M_{2}
$$

(with respect to $\mathscr{C}_{\text {ord }}$ ) is quasi-isomorphic to the total complex of the chain tricomplex $M_{1} \otimes R^{\otimes \bullet} \otimes M_{2}$, in which the three differentials are those of $M_{1}$ and $M_{2}$ plus the differential of the bar construction for $R$ as a $K$-algebra. This tricomplex can also be written $M_{1} \otimes R^{\otimes(\bullet+1)} \otimes_{R} M_{2}$. But $M_{1} \otimes R^{\otimes(\bullet+1)}$ is $R$-flat because $R^{\otimes \bullet}$ and (without loss of generality) $M_{1}$ are $K$-flat. So the total complex of the bicomplex $M_{1} \otimes R^{\otimes(\bullet+1)}$ is a flat resolution of $M_{1}$. Therefore the classical derived tensor product of $M_{1}$ and $M_{2}$ can be represented by $M_{1} \otimes R^{\otimes(\bullet+1)} \otimes_{R} M_{2}$, and therefore by $|\mathscr{M}|_{\text {ord }}$.

To complete the case $r=2$ it now suffices to prove that the $\mathbb{S}^{2}$-realization $|\mathscr{M}|$, which we defined in $(3 \cdot 5)$, is equivalent to the ordered realization $|\mathscr{M}|_{\text {ord }}$. There is certainly a natural map $|\mathscr{M}|_{\text {ord }} \rightarrow|\mathscr{M}|$, induced by inclusion of operads. This map respects skeleta, so induces a map of homology spectral sequences. We have to calculate the $E^{1}$-term in the target spectral sequence. Now the homology modulo its boundary of the complex of trees with labels $\left\{0_{1}, 1,2, \ldots, n, 0_{2}\right\}$ is the tree representation, which restricts to the regular representation of $\Sigma_{n}[23]$; and the inclusion of the ordered trees induces a map which takes the homology generator to a generator of this regular module [22]. After taking $\Sigma_{n}$-covariants as the construction of $|\mathscr{M}|$ requires, we therefore have an isomorphism of $E^{2}$-terms. Thus the spectral sequences are isomorphic, and so $|\mathscr{M}|_{\text {ord }} \rightarrow|\mathscr{M}|$ is a quasi-isomorphism. Combining this with the first result of the proof shows that $|\mathscr{M}|$ is quasi-isomorphic to $M_{1} \otimes_{R} M_{2}$. The result is now proved for $r=2$.

The proof for $r>2$ follows exactly the same lines. The only difference is the 
inclusion of a counting argument to match the numbers of generators in the free modules involved in the two $E^{2}$-terms, for these no longer have rank one. We omit the details.

The following acyclicity lemma is central to the results of the present section.

Lemma 3.7. Let $K$ be a commutative ring, and $M$ a $K$-module. Then the complex $\mathscr{K}_{K}(K ; M)$ is acyclic.

Contemplating configuration spaces makes one think that Lemma $3 \cdot 7$ should be true, but the only proof we know is combinatorial and lengthy. This proof is given in the Appendix. The first consequence of the lemma is that one can calculate $\Gamma$-homology relative to the ground ring without normalizing by quotienting by $\mathscr{K}_{K}(K ; M)$.

Proposition 3.8. Let $A$ be an $E_{\infty}$-algebra over the ground ring $K$, and $M$ an $A$ module. Then

$$
H \Gamma_{*}(A / K ; M) \approx H_{*}\left(\mathscr{K}_{K}(A ; M)\right) .
$$

Proof. Since quotienting by the acyclic complex $\mathscr{K}_{K}(K ; M)$ is a quasi-isomorphism, we have $\mathscr{K}(A / K ; M) \simeq \mathscr{K}_{K}(A ; M)$.

Naturally, one would like to be able to describe $H \Gamma(B / A ; M)$ in an equally simple way for any $E_{\infty}$ pair of algebras $A \subset B$. The tensor powers of $A$ would have to be replaced by derived tensor powers of $B$ over $A$. We have little doubt that this could be done, but the resulting elegant statement might not justify the technical mischief with derived powers which would be needed to prove the result and, later, to apply it. The following theorem is a good substitute.

Theorem 3.9 (Flat base-change for $\mathscr{K}(B / A ; M)$ ). Let $K$ be a commutative ring, and $R$ a flat commutative $K$-algebra. Then:

(1) for every $E_{\infty}$ algebra $A$ and every $A$-module $M$ which are flat over $R$, there is a quasi-isomorphism

$$
\mathscr{K}_{R}(A ; M) \simeq \mathscr{K}_{K}(A ; M) / \mathscr{K}_{K}(R ; M) ;
$$

(2) if $A$ is a subalgebra of the $E_{\infty}$ algebra $B$ and $M$ is a $B$-module, all these being $R$-flat, then the quasi-isomorphism type of $\mathscr{K}(B / A ; M)$ is the same, whether the ground ring be taken to be $K$ or $R$.

Proof. (1) Suppose $L$ is a commutative ring such that $K \subset L \subset R$. In the application, $L$ will actually be either $K$ or $R$. As in (3.1), no generality is lost by assuming that $R$ is a (strictly commutative) subalgebra of the $E_{\infty}$ algebra $A$. We have defined $\mathscr{K}_{L}(A ; M)$ as the realization of a certain $\mathscr{C}$-complex $\mathscr{K}$. This means that $|\mathscr{K}|^{\prime}$ is defined first as a certain quotient of $\bigoplus_{n \geqslant 2} \mathscr{C}_{n+1} \otimes_{\Sigma_{n}} A^{\otimes n} \otimes M$, then $\mathscr{K}_{L}(A ; M)=|\mathscr{K}|$ is constructed as the cofibre of a map $|\mathscr{K}|^{\prime} \rightarrow A \otimes M$, all tensor products being over $L$.

We construct a filtration of $|\mathscr{K}|^{\prime}$ and $A \otimes M$, and therefore of $\mathscr{K}_{L}(A ; M)$, by defining the $p$ th filtration stage $F^{p} \mathscr{K}_{L}(A ; M)$ to be the image of the submodule in which at most $p$ of the tensor factors from $A$ lie outside $R$. This respects all necessary identifications, and is thus a valid definition of a filtration in which

$$
\mathscr{K}_{L}(R ; M)=F^{0} \subset F^{1} \subset \cdots \subset F^{p-1} \subset F^{p} \subset \cdots \subset F^{\infty}=\mathscr{K}_{L}(A ; M) .
$$


Let us consider the quotient $F^{p} / F^{p-1}$. Under the action of $\Sigma_{n}$, every tensor $a_{1} \otimes \cdots \otimes$ $a_{n} \otimes m$ with $p$ factors outside $R$ is equivalent to an element in which only $a_{1}, \ldots, a_{p}$ are outside $R$; and modulo lower filtrations this element is unique up to the action of $\Sigma_{p} \times \Sigma_{n-p}$. Provided that $R$ is $L$-flat, and $p \geqslant 1$, it follows from Proposition 3.6 that $F^{p} / F^{p-1}$ is quasi-isomorphic to $E \Sigma_{p} \otimes_{\Sigma_{p}}(A / R) \otimes_{R}(A / R) \otimes_{R} \cdots \otimes_{R}(A / R) \otimes_{R} M$, where there are $p$ factors $A / R$. Now this is quite independent of $L$. So if we take the natural filtered map between the two filtered complexes

$$
\mathscr{K}_{K}(A ; M) \longrightarrow \mathscr{K}_{R}(A ; M)
$$

associated with the two choices $L=K$ and $L=R$, we know that it induces equivalences of filtration quotients $F^{p} / F^{p-1}$ for all $p \geqslant 1$. Therefore the map

$$
\mathscr{K}_{K}(A ; M) / F^{0} \mathscr{K}_{K}(A ; M) \longrightarrow \mathscr{K}_{R}(A ; M) / F^{0} \mathscr{K}_{R}(A ; M)
$$

is a quasi-isomorphism. But $F^{0} \mathscr{K}_{K}(A ; M)=\mathscr{K}_{K}(R ; M)$, and $F^{0} \mathscr{K}_{R}(A ; M)=$ $\mathscr{K}_{R}(R ; M)$ which is acyclic by Lemma $3 \cdot 7$, so this relation is precisely (1) of the statement.

(2) When $A$ is a subalgebra of the $E_{\infty}$ algebra $B$ and $M$ is a $B$-module, all these being $R$-flat, we have a diagram

$$
\begin{aligned}
& \mathscr{K}_{K}(R ; M) \stackrel{\approx}{\longrightarrow} \mathscr{K}_{K}(R ; M) \\
& \stackrel{\downarrow}{\downarrow} \mathscr{K}_{K}(A ; M) \longrightarrow \mathscr{K}_{K}(B ; M) \longrightarrow \mathscr{K}_{1}(B / A ; M) \\
& \mathscr{K}_{R}(A ; M) \longrightarrow \mathscr{K}_{R}(B ; M) \longrightarrow \mathscr{K}_{2}(B / A ; M)
\end{aligned}
$$

in which two columns are cofibrations by (1) above, and two rows are cofibrations by definition. The diagram implies that the vertical map on the right between the two models for $\mathscr{K}(B / A ; M)$ is a quasi-isomorphism.

\section{3·10. Cyclic $\Gamma$-homology and cohomology}

Let $A$ be an algebra over the cofibrant cyclic $E_{\infty}$ operad $\mathscr{C}$, with $K$ as ground ring. We then have the cyclic $\mathscr{C}$-complex $|\mathscr{M}|$ described in Exercise $2 \cdot 5$, which has $\mathscr{M}_{S}=A^{\otimes S}$, with structural maps induced by the multiplication in $A$. We denote the cyclic realization of $|\mathscr{M}|$ by $\mathscr{K}^{\mathrm{cy}}(A)$.

The cyclic $\Gamma$-homology and cyclic $\Gamma$-cohomology are defined in terms of the cyclic realization $\mathscr{K}^{\mathrm{cy}}(A)$ :

$$
\begin{gathered}
H \Gamma_{*}^{\mathrm{cy}}(A)=H_{*}\left(\mathscr{K}^{\mathrm{cy}}(A)\right) \\
H \Gamma_{\mathrm{cy}}^{*}(A)=H^{*}\left(\operatorname{Hom}_{K}\left(\mathscr{K}^{\mathrm{cy}}(A), K\right)\right) .
\end{gathered}
$$

These groups lead to topics outside the scope of this paper.

\section{Free $E_{\infty}$-algebras and the Miller spectral sequence}

Let $X$ be a chain complex of flat $K$-modules. The free $\mathscr{C}$-algebra generated by $X$ is

$$
F X=X \oplus \bigoplus_{n \geqslant 2} \mathscr{C}_{n+1} \otimes_{\Sigma_{n}} X^{\otimes n}
$$


the action of $\mathscr{C}$ being given by composition in the operad. This is a model for the free $E_{\infty}$-algebra generated by $X$. Standard methods show that up to quasi-isomorphism it depends only upon the quasi-isomorphism type of $X$, and that it is independent of the choice of the cofibrant cyclic $E_{\infty}$ operad $\mathscr{C}$.

Let $M$ be any $F X$-module. The cotangent complex $\mathscr{K}_{K}(F X ; M)$ has a particularly simple form.

Proposition $4 \cdot 1$. There is an equivalence

$$
\mathscr{K}_{K}(F X ; M) \simeq X \otimes M
$$

and the $\Gamma$-homology of the free $E_{\infty}$-ring $F X$ is therefore isomorphic to the homology of the chain complex $X$.

Proof. As always, $\mathscr{K}_{K}(F X, M)$ is the cofibre of $\varepsilon:|\mathscr{K}|^{\prime} \rightarrow F X \otimes M$, where $|\mathscr{K}|^{\prime}$ is the quotient of

$$
\bigoplus_{n \geqslant 2} \mathscr{C}_{n+1} \otimes_{\Sigma_{n}}(F X)^{\otimes n} \otimes M
$$

by the relations specified in $(2 \cdot 8)$. So we first calculate $|\mathscr{K}|^{\prime}$.

There is a natural map

$$
\alpha: \bigoplus_{n \geqslant 2} \mathscr{C}_{n+1} \otimes_{\Sigma_{n}} X^{\otimes n} \otimes M \longrightarrow|\mathscr{K}|^{\prime}
$$

induced by the inclusion of $X$ in $F X$. In fact, $\alpha$ is an isomorphism. We shall describe a two-sided inverse. Let us take a general element $f=c \otimes_{\Sigma_{n}} \gamma_{1} \otimes \cdots \otimes \gamma_{n} \otimes m$ of $|\mathscr{K}|^{\prime}$, in which we may assume $\gamma_{i}=c_{i} \otimes y_{i} \in \mathscr{C}_{r_{i}+1} \otimes_{\Sigma_{r_{i}}} X^{\otimes r_{i}}$ for $1 \leqslant i \leqslant j$, and $\gamma_{i}=x_{i} \in X$ for $j<i \leqslant n$. We can form the composite in the operad $\mathscr{C}$ of the elements $c_{i} \in \mathscr{C}_{r_{i}+1}$ with $c \in \mathscr{C}_{n+1}$, which yields an element $\bar{c} \in \mathscr{C}_{N}$, where $N=r_{1}+\cdots+r_{j}-j+n+1$. The relations $(2 \cdot 8)$ tell us that for any such $(j+1)$-fold composite in $\mathscr{C}$ we can decompose the element

$$
\bar{f}=\bar{c} \otimes_{\Sigma_{N}} y_{1} \otimes \cdots \otimes y_{j} \otimes x_{j+1} \otimes \cdots \otimes x_{n} \otimes m
$$

in a standard way as a sum of terms: the first (corresponding to $c$ in the operadic composition) is our original element $f$, and the other terms (corresponding to $\left.c_{1}, \ldots, c_{j}\right)$ are evidently in the form $\alpha\left(g_{i}\right)$ for certain well defined elements $g_{1}, \ldots, g_{j}$ in $\bigoplus_{n \geqslant 2} \mathscr{C}_{n+1} \otimes_{\Sigma_{n}} X^{\otimes n} \otimes M$. Since $\bar{f}$ is also of the form $\alpha\left(g_{0}\right)$, rearranging the formula shows that $f=\alpha\left(g_{0}-\sum_{i=1}^{j} g_{i}\right)$. The homomorphism $\beta$ given by $\beta(f)=g_{0}-\sum_{i=1}^{m} g_{i}$ respects the relations $(2 \cdot 8)$ (as one immediately verifies) and gives a two-sided inverse for $\alpha$.

Having thus identified $|\mathscr{K}|^{\prime}$, we must find the cofibre $\mathscr{K}_{K}(F X, M)$ of

$$
\varepsilon: \bigoplus_{n \geqslant 2} \mathscr{C}_{n+1} \otimes_{\Sigma_{n}} X^{\otimes n} \otimes M \longrightarrow F X \otimes M .
$$

By construction of $F X$, the target $F X \otimes M$ of $\varepsilon$ is just the sum of the source of $\varepsilon$ with $X \otimes M$. Furthermore, the formula (2.8) for $\varepsilon$ shows that the component into the first summand of the target is the identity map. Therefore the cofibre of $\varepsilon$ is isomorphic to the mapping cylinder of a certain homomorphism $|\mathscr{K}|^{\prime} \rightarrow X \otimes M$ (namely the second component of the homomorphism $\varepsilon$ ). Thus it is homotopy equivalent to $X \otimes M$ as the proposition claims. 
As a further example, let us consider the quotient of the free algebra $F X$ obtained by imposing the relation that the square of the operad should act by zero. For our cofibrant operads, the image of the operadic composition is the boundary, so the algebra which we are now considering is

$$
G X=X \oplus \bigoplus_{n \geqslant 2}\left(\mathscr{C}_{n+1} / \partial \mathscr{C}_{n+1}\right) \otimes_{\Sigma_{n}} X^{\otimes n}
$$

For the sake of simplicity we shall consider only trivial $G X$-modules $M$ (that is, we assume $X M=0$ ). We shall need the following notation. The boundary of the boundary $\partial^{2} \mathscr{C}_{n+1}$ consists of all triple products in $\mathscr{C}_{n+1}$. It is the sum of two submodules: one, denoted by $\partial_{+}^{2} \mathscr{C}_{n+1}$, in which the distinguished label 0 (linked to $M$ ) belongs to the middle factor; and the other, denoted by $\partial_{-}^{2} \mathscr{C}_{n+1}$ in which 0 belongs to one of the end factors.

Proposition 4.2. If $M$ is any trivial $G X$-module then

$$
\mathscr{K}_{K}(G X ; M) \simeq\left(X \oplus \bigoplus_{n \geqslant 3} \frac{\partial \mathscr{C}_{n+1}}{\partial_{-}^{2} \mathscr{C}_{n+1}}[-1] \otimes_{\Sigma_{n}} X^{\otimes n}\right) \otimes M
$$

Proof. We proceed as in the proof of Proposition $4 \cdot 1$. In the present case the natural map

$$
\alpha: \bigoplus_{n \geqslant 2} \mathscr{C}_{n+1} \otimes_{\Sigma_{n}} X^{\otimes n} \otimes M \longrightarrow|\mathscr{K}|^{\prime}
$$

is no longer injective, because of the relations in $G X$ : in fact its kernel consists of those terms involving $\partial_{-}^{2} \mathscr{C}_{n+1}$. The construction used for the inverse $\beta$ no longer gives uniquely-determined elements, but it does show that $\alpha$ is surjective, and therefore that

$$
|\mathscr{K}|^{\prime} \approx \bigoplus_{n \geqslant 2}\left(\frac{\mathscr{C}_{n+1}}{\partial_{-}^{2} \mathscr{C}_{n+1}} \otimes_{\Sigma_{n}} X^{\otimes n} \otimes M\right)
$$

Now $\mathscr{K}_{K}(G X ; M)$ is the cofibre of $\varepsilon:|\mathscr{K}|^{\prime} \rightarrow G X \otimes M$, which by the above we can now write as

$$
\bigoplus_{n \geqslant 2}\left(\frac{\mathscr{C}_{n+1}}{\partial_{-}^{2} \mathscr{C}_{n+1}} \otimes_{\Sigma_{n}} X^{\otimes n} \otimes M\right) \longrightarrow X \otimes M \oplus\left(\bigoplus_{n \geqslant 2} \frac{\mathscr{C}_{n+1}}{\partial \mathscr{C}_{n+1}} \otimes_{\Sigma_{n}} X^{\otimes n} \otimes M\right) .
$$

From the hypothesis that the action on $M$ is trivial, it follows that this is simply the homomorphism into the second summand induced by the inclusion $\partial_{-}^{2} \mathscr{C}_{n+1} \rightarrow \partial \mathscr{C}_{n+1}$. On the summand $n=2$ this is actually an isomorphism. The result follows.

The algebra homomorphism $F X \rightarrow G X$ induces the inclusion of the first summand in the formula given by Proposition 4.2. Applying the Transitivity Theorem of $3 \cdot 4$, we deduce the following.

Corollary $4 \cdot 3$. When $M$ is a $G X$-module with trivial action

$$
\mathscr{K}(G X / F X ; M) \simeq\left(\bigoplus_{n \geqslant 3} \frac{\partial \mathscr{C}_{n+1}}{\partial_{-}^{2} \mathscr{C}_{n+1}}[-1] \otimes_{\Sigma_{n}} X^{\otimes n}\right) \otimes M .
$$

An analysis of $\partial_{-}^{2} \mathscr{C}_{n+1}$ can now be used to reduce the determination of the relative gamma homology to a calculation in the homology of symmetric groups. 


\subsection{The Miller spectral sequence}

We can make more systematic application of free algebras by using the cotriple associated with $\mathscr{C}$. This is formed from the forgetful functor from $\mathscr{C}$-algebras to graded $K$-modules and its left adjoint the free algebra functor $F$ of Proposition 4·1. We omit any notation for the forgetful functor, and denote the cotriple itself by $F$.

Let $A$ be any $E_{\infty}$ algebra over the ground ring $K$, with structural operad $\mathscr{C}$. The cotriple $F$ generates from $A$ a simplicial $\mathscr{C}$-algebra with $F^{n+1} A$ in simplicial degree $n$. When augmented by placing $A$ in degree -1 , it has an additional degeneracy which proves it contractible as a simplicial graded module. Thus $F^{*} A=\left\{F^{n+1} A\right\}_{n \geqslant 0}$ is a simplicial free resolution of $A$. The homotopy invariance of the cotangent complex implies that

$$
\mathscr{K}_{K}\left(F^{*} A ; M\right) \simeq \mathscr{K}_{K}(A ; M)
$$

for every $A$-module $M$, and therefore the simplicial filtration of the complex on the left gives a spectral sequence

$$
E_{s, t}^{1} \approx H \Gamma_{t}\left(F^{s+1} A ; M\right) \Longrightarrow H \Gamma_{s+t}(A ; M)
$$

which certainly converges if $A$ and $M$ are bounded below. Applying Proposition 4·1 to the free $\mathscr{C}$-algebras $F^{s+1} A$ yields

$$
E_{s, t}^{1} \approx H_{t}\left(F^{s} A \otimes M\right) .
$$

Under special circumstances there is a straightforward description of $E^{2}$.

Theorem 4.5. Suppose that the ground ring is the finite prime field $\mathbb{F}_{2}$, and that $A$ is the symmetric algebra generated by a vector space $V$ in degree zero. Then the spectral sequence with $\mathbb{F}_{2}$ coefficients has the form

$$
E_{s, t}^{2} \approx \operatorname{Untor}_{s, t}^{R}\left(V, \mathbb{F}_{2}\right) \Longrightarrow H \Gamma_{s+t}\left(A ; \mathbb{F}_{2}\right)
$$

where $R$ is the Dyer-Lashof algebra, and Untor denotes torsion products of unstable $R$-modules.

Proof. Proposition $4 \cdot 1$ gives us that

$$
E_{s, t}^{1} \approx H \Gamma_{t}\left(F^{s+1} A ; \mathbb{F}_{2}\right) \approx H_{t}\left(F^{s} A\right) .
$$

On the other hand $H_{*}\left(F^{*} A\right)$ is a simplicial resolution of $H_{*}(A) \approx A=S[V]$, and a standard structure theorem on the homology of extended powers $([\mathbf{6}$, theorem $5 \cdot 1])$ implies that each term $H_{*}\left(F^{s+1} A\right)$ in this resolution is a free unstable $R$-algebra. In particular, $H_{*}\left(F^{*} A\right) \rightarrow H_{*}(A)$ is a weak equivalence of free simplicial commutative algebras, and therefore has a homotopy inverse. Applying the indecomposables functor $Q$ and using the structure theorem again, we find that $Q H_{*}\left(F^{*+1} A\right)$ is a simplicial resolution of $Q A \approx V$ by free 1-allowable $R$-modules, which after application of the functor $-\otimes_{R} \mathbb{F}_{2}$ gives precisely the above $E^{1}$ term. Therefore the $E^{2}$ term is just the unstable Tor-group $\operatorname{Untor}^{R}\left(V, \mathbb{F}_{2}\right)$.

Miller [16] has completely calculated Untor groups such as those above by building upon work of Priddy. We conjecture that the spectral sequence of Theorem 4.5 collapses from $E^{2}$ onward, and that this might be proved by introducing a further 
action of the Dyer-Lashof algebra in $\Gamma$-homology. This would be related to the 'secondary Steenrod action' of [16] and to unpublished ideas of Kriz and McClure. (Added in proof: this conjecture follows from recent work of B. Richter.)

\section{The $A_{\infty}$ analogue: Hochschild and cyclic homology}

The above theory is specifically for $E_{\infty}$ structures, and is new. We now construct the precise analogue for $A_{\infty}$ (homotopy-associative) structures, and show that this just leads to a new description of the familiar Hochschild homology and cyclic homology of associative algebras.

We replace $\mathbb{S}$ with the category $\mathbb{S}^{\text {cy }}$ of cyclically-ordered finite sets and orderpreserving isomorphisms. The automorphism group of an object of $\mathbb{S}^{\text {cy }}$ is a finite cyclic group. If 0 is chosen as basepoint in an object $S^{0}$ of $\mathbb{S}$, its complement $S$ is totally ordered, and the group of automorphisms preserving the basepoint is trivial.

We redefine operads and cyclic operads for the new case, replacing the category $\mathbb{S}$ in $(1 \cdot 1)$ and $(1 \cdot 2)$ by $\mathbb{S}^{c y}$. The composition operations have the form

$$
\circ_{s, t}: \mathscr{A}_{S} \otimes \mathscr{A}_{T} \longrightarrow \mathscr{A}_{S \sqcup_{s, t} T}
$$

where $S \sqcup_{s, t} T$ has the unique cyclic ordering obtained by concatenating the total orderings on $S \backslash\{s\}$ and $T \backslash\{t\}$. We say a cyclic operad $\mathscr{A}$ is $A_{\infty}$ if $\mathscr{A}_{S}$ is contractible for each $S$, and the cyclic group $C_{S}$ acts freely on $\mathscr{A}_{S}$. Cofibrancy is defined as before. Next we introduce algebras over a cyclic $A_{\infty}$ operad $\mathscr{A}$, and modules over these algebras by analogy with $(2 \cdot 1)$. The simplest examples are associative rings and bimodules respectively. Similarly, cyclic and non-cyclic $\mathscr{A}$-complexes are defined by precise analogy with Definitions $2 \cdot 4$ and $2 \cdot 6$. The archetypes are $\mathscr{M}_{S}=A^{\otimes S}$ in the cyclic case, and $\mathscr{M}_{S^{0}}=A^{\otimes S} \otimes M$ in the non-cyclic case, where $A$ is an associative or $A_{\infty}$ algebra and $M$ an $A$-bimodule. The realizations $|\mathscr{M}|$ and $|\mathscr{M}|_{\text {cy }}$ are defined just as in $(2 \cdot 8)$ and $(2 \cdot 9)$, the category $\mathbb{S}$ being replaced everywhere by $\mathbb{S}^{\text {cy }}$ and the symmetric group $\Sigma_{n+1}$ in (2.9) by the cyclic group $C_{n+1}$.

\subsection{Homology of the $A_{\infty}$ realization}

We have the following analogue of $(2 \cdot 13)$. It is very much simpler than the $E_{\infty}$ version, because the represention $V_{p}$ is replaced the homology of the space of cyclicallyordered $p$-trees, which is free of rank 1 .

Proposition.

(1) Let $\mathscr{M}$ be a $\mathscr{A}$-complex, where $\mathscr{A}$ is an $A_{\infty}$ operad. Then there is a homology spectral sequence

$$
E_{p-1, q}^{1} \approx H_{q}\left(\mathscr{M}_{p+1}\right) \Longrightarrow H_{p+q-1}(|\mathscr{M}|) .
$$

(2) When $\mathscr{M}$ is a cyclic $\mathscr{A}$-complex the spectral sequence has the form

$$
E_{p-1, q}^{1} \approx H_{q}\left(E C_{p+1} \otimes_{C_{p+1}} \tilde{\mathscr{M}}_{p+1}\right) \Longrightarrow H_{p+q-1}\left(|\mathscr{M}|_{c y}\right)
$$

where $\tilde{\mathscr{M}}_{p+1}$ indicates that the $C_{p+1}$-module structure of $\mathscr{M}_{p+1}$ is twisted by the sign representation.

Proof. Just as in the $E_{\infty}$ case of Proposition 2.12, the spectral sequence obtained from the skeletal filtration of $|\mathscr{M}|$ or $\left|\mathscr{M}_{\text {cy }}\right|$ is independent of the particular cofibrant cyclic $A_{\infty}$ operad used in the construction. We may therefore choose the $A_{\infty}$ tree 
operad $\mathscr{T}^{\mathrm{cy}}$, which is constructed just as in $(1 \cdot 5)$, but with the category $\mathbb{S}$ replaced by the category $\mathbb{S}^{\text {cy }}$ of cyclically-ordered sets. It is shown in [22] that $H_{p-3}\left(\mathscr{T}_{p}^{\text {cy }}\right)$ is cyclic infinite and can be described as follows. We use the triangulation of $\mathscr{T}_{p}^{\text {cy }}$ as a simplicial complex given by ([20, 1.2]). A $(p-3)$-simplex corresponds to a tree shape in which there are just three edges at each node. Let $\sigma$ be a $(p-4)$-simplex of $\mathscr{T}_{p}^{\text {cy }}$, corresponding to a tree shape containing one node $\eta$ of order 4 , all other nodes having order 3 . As shown in $([\mathbf{2 0}, 1 \cdot 2])$, the node $\eta$ may be separated in three ways so that there are three $(p-3)$-simplices having $\sigma$ as a face. It is easy to see that, if $\sigma$ is cyclically labelled in the plane, then exactly two of these three choices are cyclically labelled, so that $\sigma$ is a face of exactly two cyclically labelled $(p-3)$ simplices. In [22] it is shown that orientations may be chosen for the collection of $(p-3)$-simplices given by cyclically labelled trees so as to obtain a cycle, denoted $c_{p}$; and that the induced representation of the cyclic group $C_{p+1}$ on $H_{p-3}\left(\mathscr{T}_{p}^{\mathrm{cy}}\right)$ is the sign representation. This leads to the $E^{1}$ terms given above.

Corollary $5 \cdot 2$.

(1) Let $\mathscr{M}$ be the $\mathscr{A}$-complex with $\mathscr{M}_{S^{0}}=A^{\otimes S} \otimes M$, where $A$ is an associative $K$-algebra and $M$ an A-bimodule. Then the homology of $|\mathscr{M}|$ is the Hochschild homology of $A$, with dimension shifted by one:

$$
H_{r}(|\mathscr{M}|) \approx H H_{r+1}(A ; M) \text { for } r \geqslant 0 .
$$

(2) Let $\mathscr{N}$ be the cyclic $\mathscr{A}$-complex with $\mathscr{N}_{S}=A^{\otimes S}$. Then the homology of $|\mathscr{N}|_{c y}$ is the cyclic homology of $A$, with a dimension shift:

$$
H_{r}\left(|\mathscr{N}|_{c y}\right) \approx H C_{r+1}(A) \text { for } r \geqslant 0 .
$$

Proof. (1) Since $\mathscr{M}_{p}$ is discrete, the $E^{1}$ term of the spectral sequence of (5·1(1)) collapses to the edge $E_{p-1,0}^{1}=A^{\otimes p} \otimes M$. Analysis of the identifications in $|\mathscr{M}|$ shows that $d_{p-1,0}^{1}: A^{\otimes p} \otimes M \rightarrow A^{\otimes(p-1)} \otimes M$ is the Hochschild boundary. Thus $E_{*, 0}^{1}$ is simply the standard Hochschild complex, shifted down and truncated.

(2) Using a model where $\mathbb{S}^{\text {cy }}$ has one set of each size, we have

$$
\mathscr{T}_{n}^{\mathrm{cy}} \approx C_{*}\left(C_{n+1}\right) \otimes C_{*}\left(\tilde{T}_{n}^{\mathrm{cy}}\right),
$$

where $\tilde{T}_{n}^{\text {cy }}$ is the space of planar $n$-trees, and $C_{*}\left(C_{n+1}\right)$ is the bar construction on the cyclic group which permutes the labels $\{0,1, \ldots, n\}$ of these trees. Therefore $|\mathscr{N}|_{\text {cy }}$ is a bicomplex which has $(m, k+1)$ st group

$$
\bigoplus_{n} C_{m}\left(C_{n+1}\right) \otimes C_{k}\left(\tilde{T}_{n}^{\mathrm{cy}}, T_{n}^{\mathrm{cy}}\right) \otimes A^{\otimes(n+1)},
$$

where $T_{n}^{\mathrm{cy}}$ is the boundary of $\tilde{T}_{n}^{\mathrm{cy}}$ (the fully-grown trees). We filter by $n$. Since the complex $\tilde{T}_{n}^{\text {cy }}$ is a Stasheff $(n-2)$-cell, $C_{*}\left(\tilde{T}_{n}^{\text {cy }}, T_{n}^{\text {cy }}\right)$ has only one homology group, generated by the homology class $\left[c_{n}\right]$ of the cycle denoted $c_{n}$ in [22]. Thus each filtration quotient is a bicomplex for which the second standard spectral sequence (column homology first) collapses. We conclude that the spectral sequence associated to our filtration has $E_{n, m-1}^{1} \approx H_{m}\left(C_{n+1} ; A^{\otimes(n+1)}\right)$, where the action of the cyclic group on the tensor product includes the usual sign.

On the other hand, the cyclic homology of $A$ is given by Tsygan's bicomplex. This has $A^{\otimes(n+1)}$ in the $(m, n)$ th position, and the horizontal differentials are alternately 
$T$ and $N$, the morphisms in the standard periodic resolution of the cyclic group $C_{n+1}$. Filtration by $n$ gives rise to a spectral sequence with $E_{n, m}^{1} \approx H_{m}\left(C_{n+1} ; A^{\otimes(n+1)}\right)$.

There is an equivalence from the periodic resolution to the bar resolution which takes the generator to $[N|T| \ldots|T| N \mid T]$ in even degrees, and to $[T|N| \ldots|T| N \mid T]$ in odd degrees. We use it to construct a chain map $\theta$ from Tsygan's bicomplex (with the row $n=0$ deleted) to the bicomplex representing $|\mathscr{N}|_{\mathrm{cy}}$. Explicitly, we define

$$
\theta_{m, n}: A^{\otimes n+1} \longrightarrow C_{m}\left(C_{n+1}\right) \otimes C_{n-2}\left(\tilde{T}_{n}^{\mathrm{cy}}, T_{n}^{\mathrm{cy}}\right) \otimes A^{\otimes n+1}
$$

by setting

$$
\theta_{m, n}(a)= \begin{cases}{[N|T| \ldots|N| T] \otimes c_{n} \otimes a,} & \text { for } m \text { even } \\ {[T|N| \ldots|N| T] \otimes c_{n} \otimes a,} & \text { for } m \text { odd. }\end{cases}
$$

The map $\theta$ commutes with horizontal differentials, since we began with a map of $C_{n+1}$-complexes. To prove that it commutes with vertical differentials, one needs a calculation like that which proves that Tsygan's diagram is a bicomplex, and the fact that the vertical Hochschild differential (arising from the identifications in $|\mathscr{N}|_{\text {cy }}$ ) carries $c_{n}$ to $c_{n-1}$. Finally, $\theta$ is a map of filtered bicomplexes which has bidegree $(0,-1)$ and which induces an isomorphism on the $E^{1}$ terms of the associated spectral sequences. Hence $\theta$ induces isomorphisms $H C_{r+1}(A) \approx H_{r}\left(|\mathscr{N}|_{\mathrm{cy}}\right)$.

\section{Explicit complexes in the strictly commutative case}

Let $B$ be a strictly commutative algebra which is flat over a commutative ring $A$ and let $M$ be a $B$-module. By Proposition 3.8 and Theorem $3 \cdot 9$, we may take $A$ as the ground ring in calculating $\mathscr{K}(B / A ; M)$ and $H \Gamma_{*}(B / A ; M)$. Accordingly we denote $\otimes_{A}$ simply by $\otimes$. The $\Gamma$-cotangent complex $\mathscr{K}(B / A ; M)$ is quasi-isomorphic to $\mathscr{K}_{A}(B ; M)$ by Theorem 3.9 . When constructed using the tree operad $\mathscr{T}$, this is a bicomplex

$$
C \Gamma_{p, q}(B / A ; M)=\left(C_{q+2}\left(\mathbb{S}^{1}\right) \otimes_{\mathbb{S}^{1}} C_{p-1}\left(\tilde{T}_{\bullet}, T_{\bullet}\right)\right) \otimes_{\mathbb{S}^{1}}\left(B^{\otimes 0} \otimes M\right) .
$$

Here $\bullet$ denotes a generic object of $\mathbb{S}^{1}$, and $\circ$ denotes the same object minus its basepoint. The vertical differential $d^{\prime \prime}$ of the bicomplex is the differential of the two-sided bar construction on the category $\mathbb{S}^{1}$. The horizontal differential $d^{\prime}$ is the differential in the chain complex $C_{*}\left(\tilde{T}_{\bullet}\right)$, except that chains in the boundary $T_{\bullet}$ are identified with lower skeleta by relation $(2 \cdot 8(2)$ ). (When $n=1$, the relative chain complex $C_{*}\left(\tilde{T}_{n}, T_{n}\right)$ has to be interpreted conventionally as $A$ in degree -1 .)

We can make this smaller and more explicit by replacing $\mathbb{S}^{1}$ with the model in which there is just one object $\{0,1, \ldots, k\}$ for each $k \geqslant 1$. Then one has to make many choices about how to identify an arbitrary quotient set of $\{0,1, \ldots, k\}$ with some $\{0,1, \ldots, l\}$. (See, for example, the labelling convention described in A.3 of the Appendix.) Any coherent system of choices gives a complex

$$
C \Gamma_{p, q}(B / A ; M)=\bigoplus_{k \geqslant 1}\left(C_{q+2}\left(\Sigma_{k}\right) \otimes_{\Sigma_{k}} C_{p-1}\left(\tilde{T}_{k}, T_{k}\right)\right) \otimes_{\Sigma_{k}} B^{\otimes k} \otimes M,
$$

which is quasi-isomorphic to $(6 \cdot 1)$, though the precise horizontal differential $d^{\prime}$ depends upon the choices. Once more, the vertical differential is that of the two-sided bar construction on the symmetric groups $\Sigma_{k}$. There is a dual version for cohomology when $B$ is projective. 
Since we are working in the discrete case, the subsidiary spectral sequence of $(2 \cdot 13)$ collapses to an edge and we have the following spectral sequence

$$
E_{p-1, q}^{1} \approx H_{q}\left(\Sigma_{p} ; V_{p} \otimes B^{\otimes p} \otimes M\right) \Longrightarrow H \Gamma_{p+q-1}(B / A ; M),
$$

where $V_{p}$ is the $\Sigma_{p}$-module given by the reduced homology of the tree-space $T_{p}$. When $B$ is projective, there is a dual spectral sequence in cohomology

$$
E_{1}^{p-1, q} \approx H^{q}\left(\Sigma_{p} ; V_{p} \otimes \operatorname{Hom}\left(B^{\otimes p}, M\right)\right) \Longrightarrow H \Gamma^{p+q-1}(B / A ; M) .
$$

Theorem 6.4 [22]. The edge $q=0$ of the spectral sequence above is precisely the complex used in defining the Harrison (co)homology [13] $\operatorname{Harr}_{*}(B / A ; M)$ of $B$ (with a shift in degree). Therefore there are natural transformations

$$
H \Gamma_{p-1}(B / A ; M) \longrightarrow \operatorname{Harr}_{p}(B / A ; M), \quad H \Gamma^{p-1}(B / A ; M) \longleftarrow \operatorname{Harr}^{p}(B / A ; M)
$$

when $B$ is flat (resp. projective), which are isomorphisms when B contains a field of characteristic zero.

Proof. We give the details for homology. The edge of the spectral sequence in equation $(6 \cdot 2)$ has terms:

$$
E_{p-1,0}^{1} \approx H_{0}\left(\Sigma_{p} ; V_{p} \otimes B^{\otimes p} \otimes M\right) \approx V_{p} \otimes_{\Sigma_{p}} B^{\otimes p} \otimes M .
$$

First we describe the structure of the $\Sigma_{p}$-module $V_{p}=H_{p-3}\left(T_{p}\right)$. In [20] it is shown that $T_{p}$ has the homotopy type of a wedge of $(p-1)$ ! spheres of dimension $p-3$. Thus $V_{p}$ is a free abelian group of rank $(p-1)$ !.

Let $c_{p}$ be the integral cycle formed by the cyclically-ordered trees in the plane, as described in the proof of (5.1) above; and let $s_{i, p-i}=\sum \epsilon_{\pi} \pi^{-1}$, where $\epsilon_{\pi}$ is the sign of $\pi$ and the sum is over all $(i, p-i)$-shuffles in $\Sigma_{p}$. It is shown in [22] that $s_{i, p-i} c_{p}=0$ for $1 \leqslant i \leqslant p-1$. We outline the proof, which is by induction on $p$. The result may be easily checked for small $p$. Assume the result for $q<p$ and fix $i, 1 \leqslant i \leqslant p-1$. Let $t$ be a $p$-tree corresponding to a $(p-3)$-simplex of $T_{p}$. Consider pairs $(c, \pi)$ with $c$ a cyclically labelled tree and $\pi$ an $(i, p-i)$-shuffle. We need to show that there are an even number of such pairs having $\epsilon_{\pi} \pi^{-1} c= \pm t$, exactly half with sign + . Let $x_{1}$ and $x_{2}$ be the two edges of $t$, other than the root, which meet at the entry node of the root. Let $t_{l}$ denote the part of the tree above $x_{l}$ and let $S_{l}$ be the ordered set of labels of $t_{l}$. Without loss of generality we suppose $1 \in S_{1}$. Let $k$ denote the number of elements of $S_{1}$. We consider $(i, p-i)$-shuffles $\pi$ such that $\pi t$ has cyclic labelling. Let $j_{1}$ be the number of elements in $\left\{x \in S_{1}: x \leqslant i\right\}$ and let $j_{2}=i-j_{1}$. So $1 \leqslant j_{1} \leqslant i$ and $0 \leqslant j_{2} \leqslant i-1$. An $(i, p-i)$-shuffle has at most one descent (at $\left.i\right)$. Thus $\pi_{\mid S_{l}}$ has at most one descent, after $j_{l}$ elements. Now suppose $1 \leqslant j_{1} \leqslant k-1$ and fix a choice of $\pi_{\mid S_{2}}$ making $t_{2}$ cyclic. Then the possible choices for $\pi_{\mid S_{1}}$ correspond exactly to choices of $\left(j_{1}, k-j_{1}\right)$-shuffles $\pi^{\prime}$ such that $\pi^{\prime} t_{1}$ is cyclic. By induction there are an even number of such choices, with cancelling signs. This leaves the case $j_{1}=k$. Suppose $1 \leqslant j_{2} \leqslant p-k-1$ and fix a choice of $\pi_{\mid S_{1}}$ making $t_{1}$ cyclic. Then the choices for $\pi_{\mid S_{2}}$ correspond exactly to $\left(j_{2}, p-k-j_{2}\right)$-shuffles $\pi^{\prime \prime}$ such that $\pi^{\prime \prime} t_{2}$ is cyclic. Again we use the induction hypothesis to conclude that all such terms cancel. The only remaining case is when $j_{1}=k$ and $j_{2}=0$, so that $k=i$. Here there are exactly two possible choices of $\pi$, the identity and $(12 \ldots p)^{p-k}$. Each of these gives a cyclic tree if and only if $t$ itself is cyclic, in which case the two choices give opposite signs. We conclude that all terms of $s_{i, p-i} c_{p}$ cancel. 
It follows $([23,1 \cdot 3,1 \cdot 5])$ that $\left\{\pi\left[c_{p}\right]: \pi \in \Sigma_{p-1}\right\}$ is a $\mathbb{Z}$-basis for $V_{p}$ and that the shuffle relations completely determine the $\Sigma_{p}$-module structure of $V_{p}$. Thus $V_{p} \otimes_{\Sigma_{p}}$ $B^{\otimes p}$ is isomorphic to $B^{\otimes p}$ modulo the submodule of shuffle decomposables.

It remains to identify the differential $d^{1}: E_{p, 0}^{1} \rightarrow E_{p-1,0}^{1}$. It is straightforward to check that $d^{1}\left(\left[c_{p}\right] \otimes x_{1} \otimes \cdots \otimes x_{p} \otimes m\right)=\left[c_{p-1}\right] \otimes b\left(x_{1} \otimes \cdots \otimes x_{p} \otimes m\right)$, where $b$ denotes the usual Hochschild boundary map. The edge $E_{*, 0}^{1}$ is therefore the quotient of the Hochschild complex by the shuffle decomposables, which is precisely Harrison's complex. Hence, $E_{p-1,0}^{2} \approx \operatorname{Harr}_{p}(B / A ; M)$.

The edge map of the spectral sequence gives a natural transformation

$$
H \Gamma_{p-1}(B / A ; M) \longrightarrow \operatorname{Harr}_{p}(B / A ; M) .
$$

When $B$ contains a field of characteristic zero, the higher homology of the symmetric groups is zero, so the spectral sequence collapses to the edge and the above is an isomorphism.

Proposition $6 \cdot 5$.

(1) $H \Gamma_{0}(B / A ; M) \approx \Omega_{B / A} \otimes_{B} M, \quad H \Gamma^{0}(B / A ; M) \approx \operatorname{Der}_{A}(B, M)$;

(2) $H \Gamma^{1}(B / A ; M) \approx \operatorname{Exalcom}_{A}(B, M)$.

$\left(\right.$ Here $\operatorname{Exalcom}_{A}(B, M)$, the module of infinitesimal $A$-algebra extensions of $B$ by $M$, is as defined in $[\mathbf{1 2}], 0_{I V}$, section 18.)

Proof. (1) In the bicomplex (6-1), $C \Gamma_{0,0} / d^{\prime \prime}\left(C \Gamma_{0,1}\right) \approx B \otimes M$. The image of the horizontal differential $d^{\prime}: C \Gamma_{1,0} \rightarrow C \Gamma_{0,0}$ is spanned by the usual relations for differentials of products. It follows that $H \Gamma_{0}(B / A ; M)$ is the module of Kähler differentials $\Omega_{B / A} \otimes_{B} M$. Similarly, the zeroth cohomology group is $\operatorname{Der}_{A}(B, M)$.

(2) Suppose first that $B$ is $A$-projective. In the spectral sequence $(6 \cdot 3)$ we have $E_{2}^{0,1} \approx 0, E_{2}^{1,0} \approx \operatorname{Harr}^{2}(B / A ; M)$ and so $H \Gamma^{1}(B / A ; M) \approx \operatorname{Harr}^{2}(B / A ; M)$. This is the module of $A$-split infinitesimal commutative A-algebra extensions of $B$ by $M$. Since $B$ is projective this coincides with $\operatorname{Exalcom}_{A}(B, M)$, the module of all infinitesimal $A$-algebra extensions of $B$ by $M$.

In the general case when $B$ is not $A$-projective we have to use a simplicial resolution together with properties of André-Quillen cohomology. It is a highly important fact that $\Gamma-(\mathrm{co})$ homology extends to a homotopy invariant theory for simplicial rings, the coefficients now being a simplicial module. To see this one notes that the $\Gamma$ cotangent complex $(3 \cdot 2)$ of a simplicial ring is a simplicial $d g$-module; and one simply takes the associated total complex. The cofibrancy of the operad ensures that this is a homotopy invariant of the simplicial ring. This said, we may replace the algebra $B$ by an André-Quillen resolution $P_{*}$ consisting of polynomial algebras over $A$. Filtering the $\Gamma$-cotangent complex by the simplicial degree gives a spectral sequence

$$
E_{1}^{p, q}=H \Gamma^{q}\left(P_{p} / A ; M\right) \Longrightarrow H \Gamma^{p+q}(B / A ; M) .
$$

On the edge we have $E_{1}^{p, 0}=H \Gamma^{0}\left(P_{p} / A ; M\right)=\operatorname{Der}_{A}\left(P_{p}, M\right)$ by (1), so by definition the André-Quillen cohomology $D^{p}(B / A ; M)$ is just $E_{2}^{p, 0}$. In particular, $E_{2}^{1,0} \approx$ Exalcom $_{A}(B, M)$. But $E_{1}^{p, 1}=0$ by the first case, since $P_{p}$ is projective and polynomial. The spectral sequence now gives the result.

Corollary $6 \cdot 6$. When $B$ contains a field of characteristic zero,

$$
H \Gamma_{p}(B / A ; M) \approx D_{p}(B / A ; M), \quad H \Gamma^{p}(B / A ; M) \approx D^{p}(B / A ; M),
$$

where $D_{*}$ is André-Quillen homology. 
Proof. Again we give the details for homology. If $B$ is flat over $A$ and contains a field of characteristic zero then Harrison homology coincides with André-Quillen homology [3] so the result is given by Theorem $\mathbf{6 \cdot 4}$. If $B$ is not flat, we replace it by a simplicial André resolution by polynomial algebras, $P$. (As in the proof of Proposition $\mathbf{6} \cdot \mathbf{5}$ this is the preferred method for strictly commutative rings.) We again obtain a spectral sequence: $E_{p, q}^{1}=H \Gamma_{q}\left(P_{p} / A ; M\right) \Longrightarrow H \Gamma_{p+q}(B / A ; M)$. Since each $P_{i}$ is flat, Proposition 6.5 gives $H \Gamma_{0}\left(P_{i} / A ; M\right)=\Omega_{P_{i} / A} \otimes_{P_{i}} M$, and all higher homology groups are zero by Theorem $6 \cdot 4$. Thus the spectral sequence collapses to the edge, where $E_{*, 0}^{1}$ is exactly an André-Quillen resolution of $B$, giving the result. The case of cohomology is similar, except that 'flat' is everywhere replaced by 'projective'.

In general $\Gamma$-homology is different from André-Quillen homology and from Harrison homology. The following example shows this, and reveals a non-trivial differential in the spectral sequence in equation $(6 \cdot 2)$.

Example 6.7. First take $B=A=\mathbb{F}_{2}$. Then $1 \otimes 1 \otimes 1 \otimes 1$ is a non-bounding Harrison 4-cycle, by the calculation in ([2, section 4]). Thus $\operatorname{Harr}_{4}\left(\mathbb{F}_{2} / \mathbb{F}_{2} ; \mathbb{F}_{2}\right) \approx 0$, and by Theorem 6.4 our element $1 \otimes 1 \otimes 1 \otimes 1$ exists in $E_{3,0}^{2}$. Since Lemma $3 \cdot 7$ or the Transitivity Theorem $3 \cdot 4$ implies that $H \Gamma_{3}\left(\mathbb{F}_{2} / \mathbb{F}_{2} ; \mathbb{F}_{2}\right) \approx 0$ (and similarly for André-Quillen homology), this cycle must map by the only available differential $d^{2}$ to a non-zero element of $E_{1,1}^{2}$. (The only such element is $\alpha \otimes 1 \otimes 1$, where $\alpha$ generates $H_{1}\left(\Sigma_{2} ; \mathbb{F}_{2}\right)$; for the module $V_{2}$ is trivial). Thus $H \Gamma_{3} \approx \mathrm{Harr}_{4}$.

Now let us take $B$ to be the polynomial algebra $\mathbb{F}_{2}[X], A=\mathbb{F}_{2}, M=B /(X) \approx \mathbb{F}_{2}$. A brief calculation with shuffles shows that $E_{3,0}^{2} \approx \operatorname{Harr}_{4}\left(\mathbb{F}_{2}[X] / \mathbb{F}_{2} ; \mathbb{F}_{2}\right)$ contains no non-zero element of degree 2 in $X$. Therefore $\alpha \otimes X \otimes X \in E_{1,1}^{2}$ is an infinite cycle which is not in the image of $d^{2}$ and therefore is not a boundary. So $H \Gamma_{2}\left(\mathbb{F}_{2}[X] / \mathbb{F}_{2} ; \mathbb{F}_{2}\right) \approx 0$, and $H \Gamma_{2}$ is not André-Quillen $D_{2}$.

Theorem $6 \cdot 8$.

(1) Let $B$ and $C$ be A-algebras, with $B$ flat over $A$, and let $M$ be a $B \otimes_{A} C$-module. Then the complex $\mathscr{K}\left(B \otimes_{A} C / C ; M\right)$ is quasi-isomorphic to $\mathscr{K}(B / A ; M)$, so that

$$
H \Gamma_{*}\left(B \otimes_{A} C / C ; M\right) \approx H \Gamma_{*}(B / A ; M) .
$$

(2) Let $B$ and $C$ be flat $A$-algebras, and $M a B \otimes_{A} C$-module. Then there is a quasiisomorphism

$$
\mathscr{K}\left(B \otimes_{A} C / A ; M\right) \simeq \mathscr{K}(B / A ; M) \oplus \mathscr{K}(C / A ; M),
$$

and therefore $H \Gamma_{*}\left(B \otimes_{A} C / A ; M\right) \approx H \Gamma_{*}(B / A ; M) \oplus H \Gamma_{*}(C / A ; M)$.

(3) If $B$ is an étale $A$-algebra, then $H \Gamma_{*}(B / A ; M) \approx H \Gamma^{*}(B / A ; M) \approx 0$ for every $B$-module $M$.

Proof. (1) Since $B$ is flat over the discrete commutative ring $A$, the cotangent complex $\mathscr{K}(B / A ; M)$ is equivalent to $\mathscr{K}_{A}(B ; M)$. Also $B \otimes_{A} C$ is flat over $C$, and $\mathscr{K}\left(B \otimes_{A} C / C ; M\right)$ may be replaced by $\mathscr{K}_{C}\left(B \otimes_{A} C ; M\right)$. But standard identities with the tensor product show that $\mathscr{K}_{C}\left(B \otimes_{A} C ; M\right) \approx \mathscr{K}_{A}(B ; M)$, because these are realizations of isomorphic complexes.

(2) We have an exact triangle corresponding to the triple $A \rightarrow C \rightarrow B \otimes{ }_{A} C$

$$
\mathscr{K}(C / A ; M) \longrightarrow \mathscr{K}\left(B \otimes_{A} C / A ; M\right) \longrightarrow \mathscr{K}\left(B \otimes_{A} C / C ; M\right) .
$$


Using the quasi-isomorphism of (1) we can split this by the map

$$
\mathscr{K}(B / A ; M) \longrightarrow \mathscr{K}\left(B \otimes_{A} C / A ; M\right) .
$$

(3) The arguments of André ([1, section 20]), for the homology of a separable field extension generalize to show that this can be deduced from (1), (2) and the long exact sequence of a triple, as was observed by Quillen ([18, section 5]).

We remark that it would be unreasonable to expect Theorem $6 \cdot 8(2)$ to be true for non-discrete $E_{\infty}$ algebras, because the categorical sum is not the tensor product but the free product. The cotangent complex does appear to be additive for free products of $E_{\infty}$ algebras, though at the time of writing we have not checked the details.

It might be very profitable to investigate what the analogue of Theorem $6 \cdot 8(3)$ in the non-discrete case ought to be.

\section{A formula of Pirashvili and Richter for $\Gamma$-homology in the discrete case}

In this section we prove an elegant formula due to Pirashvili and Richter [17] which expresses the $\Gamma$-homology of a discrete algebra in terms of stable homotopy. Their paper uses our original definition of the $\Gamma$-homology of a discrete algebra, which is completely different from the definition in $(3 \cdot 2)$ above. Theorem $7 \cdot 11$ below not only proves the Pirashvili-Richter formula, but also establishes an important consistency result, showing the compatibility of our earlier work with the definition of $\Gamma$-homology which we have adopted in this paper.

As before $\mathscr{C}$ will denote a cofibrant cyclic $E_{\infty}$ operad, and $\otimes$ denotes tensor product over the ground ring $A$.

\section{7·1. Г-modules}

Following $[5,17]$ we denote by $\Gamma$ the category of based finite sets: then $\mathbb{S}^{1}$ as defined in $(1 \cdot 1)$ is the subcategory of isomorphisms in $\Gamma$. A typical object of $\Gamma$ is $T^{0}$, in which 0 denotes the basepoint and $T$ the complement of 0 .

A left $\Gamma$-module is a functor $F$ from $\Gamma$ to the category $A$-mod of modules over the ground ring. Left $\Gamma$-modules (and natural transformations of these) form an abelian category $\Gamma$-mod with sufficiently many projectives. (We consider explicit projective generators in Lemma $7 \cdot 7$ below.)

The main example we consider is the following. Let $B$ be a flat (discrete) commutative $A$-algebra, and $M$ a $B$-module. Then we have the left $\Gamma$-module $B^{\otimes \circ} \otimes M$ given by

$$
F\left(T^{0}\right)=B^{\otimes T} \otimes M
$$

for each based finite set $T^{0}=T \sqcup\{0\}$, where the morphisms of $\Gamma$ act through the algebra and module structures. This $\Gamma$-module was first studied by Loday [15], who denoted it by $\mathscr{L}(B, M)$.

\section{7·2. Three definitions of homology for $\Gamma$-modules}

We shall establish the equivalence of the following three constructions.

(1) A functor $F$ from $\Gamma$ to the category of based sets yields, by a well-known construction first described by Segal, a connective spectrum $\|F\|$ in the sense of stable homotopy theory. When $F$ is a $\Gamma$-module, this spectrum is abelian and we may take its homotopy groups $\pi_{*}\|F\|$, which are $A$-modules. 
(2) A $\Gamma$-module $F$ gives a $\mathscr{C}$-complex (also denoted $F$ ) in the sense of Definition $2 \cdot 6$. Indeed, we regard each value $F\left(S^{0}\right)$ as a trivial chain complex; the required maps $\circ_{0,1}^{*}$ and $\circ_{1,0}^{*}$ of Definition $2 \cdot 6$ are constant over the operad $\mathscr{C}$ and are simply the homomorphisms $F(S \sqcup T)^{0} \rightarrow F\left(T^{01}\right)$ and $F(S \sqcup T)^{0} \rightarrow F\left(S^{0}\right)$ induced by the corresponding morphisms of $\Gamma$, which respectively collapse $S$ to $\{1\}$ and collapse $T^{0}$ to the basepoint. We form the realization $|F|$ of this $\mathscr{C}$-complex as in $(2 \cdot 8)$, and take its homology $H_{*}|F|$.

(3) There is an explicit chain complex $C_{*}^{\Gamma}(F)$ based upon a nerve construction using the category of surjections of finite sets. We need no details of it here: we refer the reader to [17] where it is called the Robinson-Whitehouse complex, as it is a direct generalization of a construction attributed to Robinson in the thesis of Whitehouse [22]. We take the homology $H_{*}^{\Gamma}(F)$ of the complex $C_{*}^{\Gamma}(F)$.

For the case when $F$ is the $\Gamma$-module $B^{\otimes 0} \otimes M$, which takes values in $B$-mod, these three constructions give respectively:

(1) the stable homotopy $\pi_{*}\left\|B^{\otimes \circ} \otimes M\right\|$ of the corresponding spectrum;

(2) the $\Gamma$-homology $H \Gamma_{*}(B / A ; M)$ of the discrete algebra $B$ as defined in Section 3 above (see Proposition 3.8);

(3) the $\Gamma$-homology of this algebra as defined in [22].

Theorem 7.3 (Pirashvili-Richter). There is a natural isomorphism, valid for all $\Gamma$ modules $F$, between the first and third constructions in (7.2):

$$
\pi_{*}\|F\| \approx H_{*}^{\Gamma}(F) .
$$

The essence of the method which Pirashvili and Richter use to prove Theorem 7.3 is this. Both the homology groups of the chain complex $C_{*}^{\Gamma}$ and the homotopy groups of the spectrum form exact connected sequences of functors on the category of left $\Gamma$ modules. Some explicit homotopies and calculations reveal that both of them vanish in positive degrees on projective generators, and that they are naturally isomorphic in degree zero. Therefore they coincide in all degrees for all $\Gamma$-modules.

By applying these same ideas we shall extend the above result as follows.

Theоrem 7·4. The homology groups $H_{*}|F|$ given by the second construction in (7·2) are also naturally isomorphic to the groups given by the first and third constructions, for all $\Gamma$-modules $F$.

The proof of Theorem $7 \cdot 4$ is developed in $(7 \cdot 5)-(7 \cdot 10)$.

Lemma 7.5. Each of the three constructions in (7.2) forms an exact connected sequence of functors on the abelian category $\Gamma$-mod.

Proof. For the first and third constructions this is contained in the proof of $[\mathbf{1 7}$, theorem 1]. For the second construction, it follows from the cofibrancy of $\mathscr{C}$ that the realization $|F|$ is an exact functor of $F$, as one sees by using induction on skeleta. Therefore the homology $H_{*}|F|$ is an exact connected sequence.

\subsection{Projective generators and their realizations}

Let $L$ be the left $\Gamma$-module which assigns to each based finite set $T^{0}=T \sqcup\{0\}$ the $A$-module $A T^{0} / A 0$, the free $A$-module generated by the set $T^{0}$ quotiented by 
the submodule generated by 0 . Let tensor products of left $\Gamma$-modules be defined termwise, that is by

$$
(F \otimes G)\left(T^{0}\right)=F\left(T^{0}\right) \otimes_{A} G\left(T^{0}\right),
$$

the action of $\Gamma$ being the evident diagonal one. The following result is proved in [17].

Lemma 7.7. The left $\Gamma$-modules $L^{\otimes n}$ for $n \geqslant 0$ form a set of projective generators for the abelian category of left $\Gamma$-modules.

The homotopy groups of the associated spectra are as follows. When $n=1$

$$
\pi_{i}\|L\| \approx \begin{cases}A, & \text { for } i=0 \\ 0, & \text { for } i \neq 0 .\end{cases}
$$

When $n \neq 1$, however, $\pi_{i}\left\|L^{\otimes n}\right\| \approx 0$ for all $i$.

The main technical lemma which we require states that the homology of our $E_{\infty}$ realization has identical properties.

Lemma 7.8. The homology groups of the $E_{\infty}$ realizations of the projective generators $L^{\otimes n}$ are as follows. When $n=1$

$$
H_{i}|L| \approx \begin{cases}A, & \text { for } i=0 \\ 0, & \text { for } i \neq 0 .\end{cases}
$$

When $n \neq 1$, however, $H_{i}\left|L^{\otimes n}\right| \approx 0$ for all $i$.

Proof. We first dispose of the technically difficult case $n=0$. The zeroth tensor power of $L$ is the $\Gamma$-module with the constant value $A$, so that the realization $\left|L^{0}\right|$ is precisely $\mathscr{K}_{A}(A ; A)$ (in the notation of Section 3 ). The acyclicity of this complex is Lemma 3.7, which is proved by an explicit combinatorial argument in the Appendix.

The remaining cases $n \geqslant 1$ will now be proved by the use of explicit homotopies, which fortunately are easier to construct than that used when $n=0$. First note that we may construct the realization $\left|L^{\otimes n}\right|$ by using any cofibrant cyclic $E_{\infty}$ operad. We again choose the tree operad of (1.5); some details of labelling and ordering conventions are described in the Appendix. As always we first construct a complex $\left|L^{\otimes n}\right|^{\prime}$ : in the present case a base for the chains consists of elements

$$
\rho \otimes\left[\sigma_{1}\left|\sigma_{2}\right| \ldots \mid \sigma_{k}\right] \otimes t
$$

in degree $k+\operatorname{dim} t$, where $t$ is a standard oriented cube in the cell structure of the tree space $\tilde{T}_{m}$ for some $m \geqslant 2$, the $\sigma_{i}$ are permutations in $\Sigma_{m}$, and $\rho:[n] \rightarrow[m]$ is a based map. Since $\rho$ represents the tensor factors in $L^{\otimes n}$, the chain is zero if $0 \in \operatorname{im} \rho$. The boundary in the chain complex is the sum of a vertical differential replacing $t$ by $\partial t$ (with appropriate identifications) and a horizontal differential from the bar construction on $\Sigma_{m}$ in which the zeroth face is the right action $\sigma_{1}^{-1} \rho$ on $\operatorname{Hom}([n],[m])$ and the final face is the left action $\sigma_{k} t$ on the tree space.

In order to define our chain homotopies, we introduce a branching construction on trees in $\tilde{T}_{m}$. A cubical cell in $\tilde{T}_{m}$ corresponds to a tree $t$ (without edge-lengths) having a root labelled 0 and leaves labelled 1 to $m$. Let $z$ be any edge of $t$. We form an $(m+1)$-tree $Y_{z}(t)$, corresponding to a cube of dimension one greater in $\tilde{T}_{m+1}$, by sprouting a new leaf labelled $m+1$ from the midpoint of the edge $z$. 
Still assuming $n \geqslant 1$, we now define a chain homotopy $\Xi$ on $\left|L^{\otimes n}\right|^{\prime}$ by giving its values on basis elements. If $\alpha$ is the chain (7.9) above, let $r \in[m]$ be the label $\left(\sigma_{1} \ldots \sigma_{k}\right)^{-1} \rho(n)$ on the tree $t$, obtained by tracing the image $\rho(n)$ through all the permutations; and let $x_{1}, \ldots, x_{s}$ be the sequence of edges of $t$ forming the shortest arc from the leaf labelled $r$ to the root. We set

$$
\Xi(\alpha)=\hat{\rho} \otimes\left[\bar{\sigma}_{1}\left|\bar{\sigma}_{2}\right| \ldots \mid \bar{\sigma}_{k}\right] \otimes \sum_{i=1}^{s} \varepsilon_{i} Y_{x_{i}}(t),
$$

where $\hat{\rho}:[n] \rightarrow[m+1]$ is defined by

$$
\hat{\rho}(i)= \begin{cases}\rho(i) & \text { if } i \leqslant n-1, \\ m+1 & \text { if } i=n,\end{cases}
$$

$\bar{\sigma}_{j}$ is the image of $\sigma_{j}$ under the natural inclusion $\Sigma_{m} \rightarrow \Sigma_{m+1}$; the signs $\varepsilon_{i}= \pm 1$ are chosen in accordance with the ordering convention of the Appendix so that the common face of the cubes $Y_{x_{i}}(t)$ and $Y_{x_{i+1}}(t)$ (in which the new leaf is attached at the vertex between $x_{i}$ and $\left.x_{i+1}\right)$ cancels in $\partial \Xi(\alpha)$.

It is clear that $\Xi$ is equivariant with respect to the symmetric group acting by switching labels, so that $\Xi$ is well defined.

A straightforward but tedious calculation shows that when $n=1$

$$
\partial \Xi+\Xi \partial=1
$$

so that the homology of $|L|^{\prime}$ is zero in all degrees. When $n>1$, with $\alpha$ as in (7.9), there is an extra uncancelled term in $\partial \Xi(\alpha)$ when $t$ is a star-tree (that is, $t$ has no internal edges). In fact

$$
(\partial \Xi+\Xi \partial) \alpha= \begin{cases}\alpha & \text { if } t \text { has internal edges, } \\ \xi \otimes[1|1| \ldots \mid 1] \otimes \star_{2} & \text { if } t \text { is a star-tree, }\end{cases}
$$

where $\star_{2}$ is the star-tree labelled by $[2]=\{0,1,2\}$, the permutations occurring in the bar construction are all trivial, and $\xi:[n] \rightarrow[2]$ is the based map defined by

$$
\xi(i)= \begin{cases}1 & \text { if } 1 \leqslant i \leqslant n-1 \\ 2 & \text { if } i=n .\end{cases}
$$

Therefore $\left|L^{\otimes n}\right|^{\prime}$ has the homology of a point when $n>1$.

As always, the $E_{\infty}$ realization $\left|L^{\otimes n}\right|$ is the cofibre of a map $\varepsilon:\left|L^{\otimes n}\right|^{\prime} \rightarrow L^{\otimes n}[1]$. Since $L^{\otimes n}[1]$ is a point in every case, and $\varepsilon$ induces an isomorphism of homology when $n>1$, the homology of $\left|L^{\otimes n}\right|$ is as stated in the lemma.

7·10. Proof of Theorem $7 \cdot 4$. The category of left $\Gamma$-modules, or functors $F: \Gamma \rightarrow$ $A$-mod, is an abelian category with sufficiently many projective objects. By Lemma $7 \cdot 5$ the homology groups $H_{i}|F|$ of the $E_{\infty}$ realization $|F|$, like the spectral homotopy groups $\pi_{i}\|F\|$ considered by Pirashvili and Richter, form a connected exact sequence of functors on this category $\Gamma$-mod. Both these sets of functors are zero for $i<0$. By Lemmas 7.7 and 7.8 they are isomorphic in degree zero on a set of projective generators for $\Gamma$-mod, and they both vanish on those generators in all degrees $i>0$. By a standard argument, the two types of functors coincide in every degree on all objects. Thus there are natural isomorphisms $H_{i}|F| \approx \pi_{i}\|F\|$ (commuting with connecting homomorphisms) for all $i$ and $F$, and Theorem $7 \cdot 4$ is proved. 
Applying Theorem $7 \cdot 4$ to the $\Gamma$-module $B^{\otimes 0} \otimes M$ we obtain the following immediate corollary.

Theorem 7-11. Let $B$ be a flat commutative A-algebra and $M$ a $B$-module. Then the following are all naturally isomorphic:

(1) the $\Gamma$-homology $H \Gamma_{i}(B / A ; M)$ as defined in $(3 \cdot 2)$;

(2) the homology $H_{i}^{\Gamma}(B / A ; M)$ of the explicit chain complex described in [17, 22];

(3) the homotopy $\pi_{i}\left\|B^{\otimes \circ} \otimes M\right\|$ of the spectrum associated with the $\Gamma$-module of Loday. The natural isomorphisms commute with connecting homomorphisms where appropriate.

$$
\text { Appendix. Acyclicity of } \mathscr{K}_{A}(A ; M)
$$

\section{A. Contraction of a certain complex without permutations}

We construct, then contract, a certain chain complex related to $\mathscr{K}_{A}(A ; M)$. It is obtained by glueing together the chains on the various tree spaces $\tilde{T}_{n}$, for $n \geqslant 2$. For simplicity we may as well take $M$ to be the ground ring $A$. The construction of our complex $K_{\infty}$ requires a labelling convention for trees, which is detailed below. The contraction requires an ordering convention for the edges of a tree. Both these conventions are somewhat arbitrary at this stage, but they have to be compatible with each other.

\section{A.1. Ordering convention}

Let $t \in \tilde{T}_{n}$ be an $n$-tree. It therefore has a root labelled 0 , and leaves labelled $1,2, \ldots, n$. Let $\beta_{i}$ be the are (shortest path) in $t$ from the leaf $i$ to the root. Then $t=\bigcup_{i=0}^{n} \beta_{i}$. We introduce a total ordering on the set of edges of $t$ as follows. If $x, y$ are edges, then $x$ precedes $y$ (written $x<y$ ) if either $x$ and $y$ are in some common $\operatorname{arc} \beta_{i}$ with $y$ nearer the root, or $\min \left\{i \mid x \in \beta_{i}\right\}>\min \left\{j \mid y \in \beta_{j}\right\}$. This does define a total ordering, in which an internal edge occurs at the first moment after all edges above it have been counted. When no internal edge is available, the next leaf (in descending order) is taken. So the leaf $n$ is, perversely, first. The root is last.

\section{A.2. The trees $t / x$ and $t \backslash x$}

An internal edge $x$ in an $n$-tree $t$ divides the tree into two. The portion including the root (and the edge $x$ itself) is a sub-tree called $t \backslash x$. The other part, containing some leaves and $x$ itself but not the root, is called the sub-tree over $x$ and is written $t / x$. It is much better to regard $t / x$ as the identification space obtained by crushing the sub-tree $t \backslash x$ to a single edge, and $t \backslash x$ as obtained by identifying $t / x$ to an edge. (If $x$ is a leaf or the root of $t$, the symbols $t / x$ and $t \backslash x$ are interpreted as either the whole of $t$ or the tree consisting of a single leaf, as appropriate.) Now we have to decide how to label these quotient trees.

\section{A·3. Labelling convention}

A quotient tree such as $t / x$ is naturally labelled by subsets forming a partition of the set $\{0,1, \ldots, n\}$, because a new leaf or root inherits all the labels on the subtree it came from. We replace these subsets by $0,1, \ldots, r$, labelling the subsets in increasing order of their minimal elements.

The point of the labelling convention is that the conventional ordering introduced above is compatible with identifying a subtree to a single edge, provided one regards a subtree as enumerated when all its edges have been enumerated. For instance, a 
subtree containing the root is always labelled 0 , and comes last in the conventional ordering.

Now we are ready to start defining our chain complex. To begin with we use reduced cubical chains, because $\tilde{T}_{n}$ is naturally a cubical complex.

Definition A.4. Let $K_{\infty}^{\prime}$ be $\bigcup_{n=2}^{\infty} K_{n}^{\prime}$, where the complexes $K_{n}^{\prime}$ are defined inductively as follows:

(1) $K_{2}^{\prime}$ is the chain complex $C_{*}\left(\tilde{T}_{2}\right)$ of the one-point tree space $\tilde{T}_{2}$.

(2) For $n \geqslant 3$, suppose that we have already defined the complex $K_{n-1}^{\prime}$ as a quotient of $\bigoplus_{2 \leqslant i<n} C_{*}\left(\tilde{T}_{i}\right)$. Then the complex $K_{n}^{\prime}$ is obtained by attaching $C_{*}\left(\tilde{T}_{n}\right)$ to $K_{n-1}^{\prime}$ along the subcomplex $C_{*}\left(T_{n}\right)$ of fully-grown trees. The attaching map $\varphi_{n-1}: C_{*}\left(T_{n}\right) \rightarrow K_{n-1}^{\prime}$ takes the generator corresponding to an $n$-tree $t$ with fully-grown edge $x$ to the class $(-1)^{n-r} t / x+(-1)^{r+1} t \backslash x$ in $K_{n-1}^{\prime}$, where $r$ is the number of leaves in $t / x$.

The previous identifications in $K_{n-1}^{\prime}$ ensure that the attaching map is well-defined and independent of the choice of the edge $x$, and evidently $K_{n}$ is by construction a quotient of $\bigoplus_{2 \leqslant i \leqslant n} C_{*}\left(\tilde{T}_{i}\right)$. The cubes $t / x$ and $t \backslash x$ are of course labelled by the convention above, and oriented by the ordering convention. It should be noted that whenever $t$ has more than one internal edge, at least one of the cubes $t / x$ and $t \backslash x$ is a degenerate face.

\section{A.5. Subdividing $K_{\infty}^{\prime}$}

We shall show that there is a natural, geometrically-inspired contraction of the complex $K_{\infty}^{\prime}$. It is not easy to describe in terms of the cubical chains, because geometrically the image of $\tilde{T}_{n}$ is deformed through $\tilde{T}_{2 n}$ in a way which is not cellular, but diagonal, on the cubes.

Therefore we replace each cubical complex $\tilde{T}_{n}$ by its natural simplicial subdivision, in which each $r$-cube is replaced by $r$ ! $r$-simplices. (An $n$-tree belongs to one or other of these, depending upon which internal edges are longer than which others. Diagonal simplices in $\tilde{T}_{n}$ contain trees having certain edges of equal length.) Every cubical chain is a chain of the simplicial subdivision, so we have enlarged $C_{*}\left(\tilde{T}_{n}\right)$; and we make identifications among these exactly as before to obtain a chain complex $K_{\infty}$, quasi-isomorphic to and containing $K_{\infty}^{\prime}$. But we continue to use cubes as blocks of simplices (sums of generators) in $K_{\infty}$.

\section{A.6. Informal description of the contraction}

The contraction of $K_{\infty}$ closely follows this geometrical idea. A labelled $n$-tree $t$ passes through $N$ stages $t_{0}, t_{1}, \ldots, t_{N}$ during the homotopy, where $t_{0}=t$ and $N$ is the total number of edges of $t$. In the tree $t_{i}$ there are two identical copies of each of the first $i$ edges in the conventional order, and one copy of the others. As identical edges must have the same length, $t_{i}$ represents a diagonal cube in some $\tilde{T}_{n+j}$ having the same dimension as $t$. The homotopy connecting $t_{i-1}$ and $t_{i}$ is represented by a tree $\Delta_{i}$ like $t_{i}$ but with one new edge below the two copies of the $i$ th edge, connecting the most recently-doubled edge to the undoubled part. This is a cube of dimension one higher. Shrinking one undoubled edge, or two identical edges, to a point is a cubical face operator: therefore $\Delta_{i}$ has $t_{i-1}$ and $t_{i}$ as faces. Finally, $t_{N}$ is the sum of two copies of $t$. A more formal description follows. 

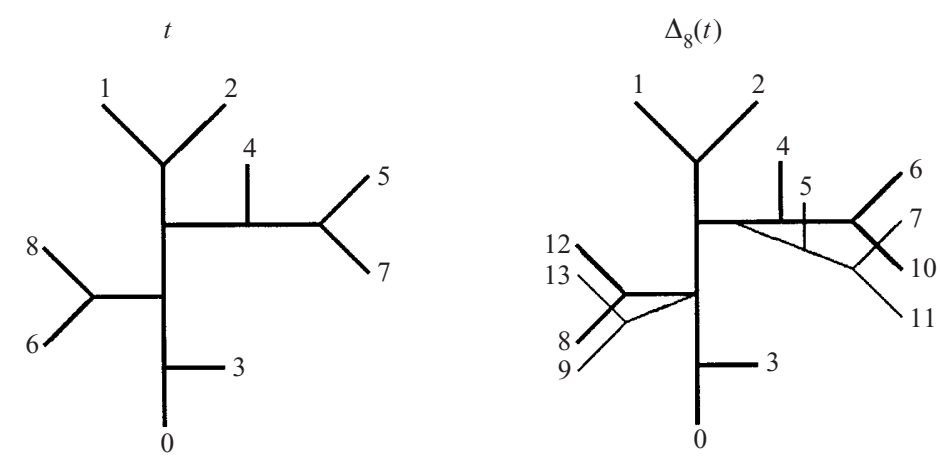

Fig. 1. Example of $\Delta_{i}(t)$.

\section{A.7. The double of a tree}

Let $t$ be an $n$-tree. The double $Y(t)$ is the $2 n$-tree obtained by taking two identical copies $t^{\prime}$ and $t^{\prime \prime}$ of the tree $t$, and grafting them by the roots onto the two leaves of the unique tree in $\tilde{T}_{2}$. Pairs of identical edges have the same length. We label the result as follows. The two leaves formerly labelled $i$ are marked $i-\frac{1}{2}$ and $i$ in $t^{\prime}$ and $t^{\prime \prime}$ respectively. Then all labels are multiplied by two to give integers.

\section{A.8. The construction $\Delta_{i}$}

We actually define $\Delta_{i}(t)$ and $t_{i}$ by induction on $i$. We set $t_{0}=t$. If $t_{i-1}$ has been defined, and $x_{i}$ is the $i$ th edge of $t$ in the conventional ordering, we define $\Delta_{i}(t)$ to be the result of grafting the double $Y\left(t / x_{i}\right)$ by its root onto the leaf $x_{i}$ of $t_{i-1} \backslash x_{i}$. We define $t_{i}$ by shrinking the grafted internal edge (formerly the root of the double $\left.Y\left(t / x_{i}\right)\right)$ of $\Delta_{i}(t)$ to a point. It follows from the inductive definition that $t_{i}$ contains two copies of edges $x_{1}, \ldots, x_{i}$ and one copy of the higher-numbered edges. We note that $\Delta_{i}(t)$ and $t_{i}$ have been defined cube by cube, or a block of generators of $K_{n}$ at a time.

We have to label $t_{i}$ and $\Delta_{i}(t)$. As for the doubling construction, we give the two copies of the leaf formerly designated $i$ the labels $i-\frac{1}{2}$ and $i$, without changing the labels on the undoubled leaves. Then we replace the labels in bijective orderpreserving fashion with the integers $1,2, \ldots, s$ for some $s$.

Definition A.9. If $t$ is a cube corresponding to a tree-shape with a total of $N$ edges, we define $\Delta(t)=\sum_{i=1}^{N}(-1)^{i} \Delta_{i}(t)$.

We claim that this defines a contracting homotopy of $K_{\infty}$ by specifying it on the generating simplices, a cubical block at a time. To prove this, we must verify that $\partial \Delta+\Delta \partial=1-\pi$, where $\pi: K_{\infty} \rightarrow K_{\infty}$ factors through the chain complex of a point. So we have to investigate how $\Delta$ commutes with respect to face relations. This includes verifying that $\Delta$ respects the identifications used to define $K_{\infty}$.

As we are still working with cubical blocks in $K_{\infty}$, even though some of them may be diagonal cubes with certain coordinates equal, it is the cubical face operators we have to check. Let $x_{i}$ be the $i$ th edge of a tree $t$ corresponding to a certain cube, also denoted $t$, in $K_{\infty}$. If $x_{i}$ is an internal edge, there is a face operator $\partial_{i}$ corresponding to shrinking the length of $x_{i}$ (and of all edges forced to have the same length) to zero. When the length of $x_{i}$ stretches to 1 , we have the opposite face $\varepsilon_{i}$ of the cube, which 
by construction of $K_{\infty}$ is identified with $\pm t / x_{i} \pm t \backslash x_{i}$ (which is the zero chain when $x_{i}$ lies between two internal edges of $t$ ).

By checking the geometrical details, we can now verify a whole slew of 'cubical identities' such as (to give one instance)

$$
\varepsilon_{i} \Delta_{j}(t)= \pm t / x_{i} \pm t / x_{i}+\Delta_{j-f+1}\left(t \backslash x_{i}\right)
$$

when $x_{i}$ is an edge of $t / x_{j}$ (which implies $i \leqslant j$ ) and where $f$ denotes the number of edges of $t / x_{i}$. The enumeration of faces is more complex here than in the case of the usual simplicial or cubical identities, because of the branching of trees. But some of the formulae simply assert that a certain face is degenerate, and is therefore a zero chain. For instance, the above formula gives a non-zero right hand side only in two cases: first, when $x_{j}$ is the root of $t$ and $i=j$; second, when $x_{i}$ has nothing but leaves above it.

In calculating these identities it is essential to remember that $\partial_{i}$ affects identical edges simultaneously and not separately, and likewise for $\varepsilon_{i}$.

\section{A-10. The cubical identities in full}

Let $x_{i}$ and $x_{j}$ be edges of $t$ and let $f$ be the number of edges of $t / x_{i}$. We denote the number of free edges (leaves plus root) of a tree $s$ by $l(s)$. Then

(1) If $i \leqslant j$ and $x_{i}$ is an internal edge or the root of $t / x_{j}$

$$
\varepsilon_{i} \Delta_{j}(t)=(-1)^{l\left(\Delta_{j}(t) \backslash x_{i}\right)} t / x_{i}+(-1)^{l\left(\Delta_{j}(t) \backslash x_{i}\right)} t / x_{i}+\Delta_{j-f+1}\left(t \backslash x_{i}\right) .
$$

(2) If $i<j$ and $x_{i}$ is an internal edge of $t \backslash x_{j}$

$$
\varepsilon_{i} \Delta_{j}(t)=(-1)^{l\left(\Delta_{j}(t) \backslash x_{i}\right)} t_{j-1} / x_{i}+\Delta_{j-f+1}\left(t \backslash x_{i}\right) .
$$

(3) If $i \geqslant j, x_{i}$ is an internal edge of $t$ and $x_{j}$ is the qth edge of $t / x_{i}$

$$
\varepsilon_{i+1} \Delta_{j}(t)=(-1)^{l\left(\Delta_{j}(t) / x_{i}\right)} t_{j-1} \backslash x_{i}+(-1)^{l\left(\Delta_{j}(t) \backslash x_{i}\right)} \Delta_{q}\left(t / x_{i}\right) .
$$

(4) For all $i$ such that $x_{i}$ is a leaf of $t$

$$
\varepsilon_{i+1} \Delta_{i}(t)=-t_{i-1}+(-1)^{l\left(t_{i-1}\right)} \Delta_{1}\left(t / x_{i}\right) .
$$

(5) If $i>j, x_{i}$ is an internal edge of $t$ and $x_{j}$ is the $p$ th edge of $t \backslash x_{i}$

$$
\varepsilon_{i+1} \Delta_{j}(t)=(-1)^{l\left(\Delta_{j}(t) \backslash x_{i}\right)} t_{j-1} / x_{i}+(-1)^{l\left(\Delta_{j}(t) / x_{i}\right)} \Delta_{p}\left(t \backslash x_{i}\right) .
$$

(6) If $i<j$ and $x_{i}$ is an internal edge of $t$

$$
\partial_{i} \Delta_{j}(t)=\Delta_{j-1}\left(\partial_{i} t\right)
$$

(7) For all $i$

$$
\partial_{i} \Delta_{i}(t)=t_{i-1}=\partial_{i} \Delta_{i-1}(t) .
$$

(8) If $i>j$ and $x_{i}$ is an internal edge of $t$

$$
\partial_{i+1} \Delta_{j}(t)=\Delta_{j}\left(\partial_{i} t\right) .
$$

(9) If $i \leqslant j$

$$
\Delta_{i} \Delta_{j}=\Delta_{j+1} \Delta_{i}
$$


The first five identities, together with the labelling convention, imply that $\Delta$ is compatible with the identifications used in defining $K_{\infty}$, and is therefore well-defined. The fourth identity gives, according to the dimension of the cube $t$,

$$
\varepsilon_{2} \Delta_{1}(t)= \begin{cases}-t & \text { if } \operatorname{dim} t>0 \\ -t+(-1)^{l(t)} *_{2} & \text { if } \operatorname{dim} t=0\end{cases}
$$

where $*_{2}$ is the unique 2 -tree. The first identity gives, when $x_{N}$ is the last edge (root) of $t$

$$
\varepsilon_{N} \Delta_{N}(t)= \begin{cases}(-1)^{l(t)+1} 2 t & \text { if } \operatorname{dim} t>0 \\ (-1)^{l(t)+1} 2 t+*_{2} & \text { if } \operatorname{dim} t=0 .\end{cases}
$$

From the cubical identities, it follows that $\Delta$ is a chain homotopy from $1+\pi$, where 1 is the identity map and $\pi$ is a point map as above, to twice the identity map. (There is additional checking to be done on 0 -chains; $\pi$ is given by $\pi\left(*_{n}\right)=(-1)^{n}(n-1) *_{2}$, where $*_{n}$ denotes the star tree with $n$ leaves.) Therefore $1-\pi$ is null homotopic by the chain homotopy $\Delta$, and $K_{\infty}$ is contractible.

\section{B. Acyclicity of $\mathscr{K}_{A}(A ; M)$}

The chain complex $\mathscr{K}_{A}(A ; M)$ is constructed as the cofibre of a map from a partial realization $|\mathscr{M}|^{\prime}$ to $M$. It very easily follows that $\mathscr{K}_{A}(A ; M)$ is acyclic if $|\mathscr{M}|^{\prime}$ is contractible; so this contractibility is what we have to prove. It is sufficient to treat the case when the coefficient module $M$ is $A$.

We describe $|\mathscr{M}|^{\prime}$. Just as $K_{\infty}$ in the previous section was constructed by glueing together the tree spaces $\tilde{T}_{n}$ according to certain labelling conventions, so $|\mathscr{M}|^{\prime}$ is obtained by glueing together the spaces $\mathscr{C}_{n} / \Sigma_{n}$ of a cofibrant cyclic $E_{\infty}$ operad $\mathscr{C}$, for which we shall use the tree operad. (In the new context, it can be seen that the somewhat arbitrary labelling convention is actually quite immaterial: a different choice leads by conjugation in symmetric groups to homotopic glueing maps, and so to a quasi-isomorphic result. But a choice has to be made.)

Thus $|\mathscr{M}|^{\prime}$ is an extended version of $K_{\infty}$, incorporating the actions of the symmetric groups. One tries to contract it by applying fibrewise the contraction of $K_{\infty}$. This amounts to constructing a coherent system of higher homotopies among the contractions obtained by twisting the original contraction by all elements of the symmetric group. One expects to be able to do this since, if $\Delta$ and $\Xi$ are two contractions of a complex, then $\Xi \Delta$ is a homotopy of homotopies from $\Delta$ to $\Xi$.

\section{B.1. Construction of $|\mathscr{M}|^{\prime}$}

We construct $|\mathscr{M}|^{\prime}$ using the cofibrant tree operad $\mathscr{T}$ of $(1 \cdot 5)$. Since the symmetric group $\Sigma_{n}$ acts trivially on the $n$th tensor power of $A$ over itself, the realization is built by glueing together the complexes $\mathscr{T}_{n} / \Sigma_{n}$. The free chain complex $\mathscr{T}_{n} / \Sigma_{n}$ has generators

$$
\left[\sigma_{1}\left|\sigma_{2}\right| \ldots \mid \sigma_{k}\right] \otimes t
$$


in dimension $k+\operatorname{dim} t$, where $k \geqslant 0, \sigma_{1}, \ldots, \sigma_{k} \in \Sigma_{n}$, and $t$ is a simplex (or cube) of the tree space $\tilde{T}_{n}$. The boundary is given by

$$
\begin{aligned}
\partial\left(\left[\sigma_{1}\left|\sigma_{2}\right| \ldots \mid \sigma_{k}\right] \otimes t\right) & \\
= & {\left[\sigma_{2}|\ldots| \sigma_{k}\right] \otimes t+\sum_{j=1}^{k-1}(-1)^{j}\left[\sigma_{1}|\ldots| \sigma_{j} \sigma_{j+1}|\ldots| \sigma_{k}\right] \otimes t } \\
& +(-1)^{k}\left[\sigma_{1}|\ldots| \sigma_{k-1}\right] \otimes \sigma_{k} t+(-1)^{k+1}\left[\sigma_{1}\left|\sigma_{2}\right| \ldots \mid \sigma_{k}\right] \otimes \partial t,
\end{aligned}
$$

where $\partial t$ is the boundary in $\tilde{T}_{n}$, and $\sigma_{k} t$ is defined using the permutation action of $\Sigma_{n}$ on the labels of $\tilde{T}_{n}$.

The identifications which form $|\mathscr{M}|^{\prime}$ from the chain complexes $\mathscr{T}_{n} / \Sigma_{n}$ mirror those used to form $K_{\infty}$ from the $\tilde{T}_{n}$. In the latter case, we recall, when an internal edge $x_{j}$ of $t$ has length 1 , the chain $t$ of $K_{\infty}$ is identified with $\pm t / x_{j} \pm t \backslash x_{j}$, which are trees labelled by our convention. This labelling convention is sufficiently functorial to allow us to identify, when $x_{j}$ has length 1 , the chain $\left[\sigma_{1}|\ldots| \sigma_{k}\right] \otimes t$ with

$$
(-1)^{n-r}\left[\hat{\sigma}_{1}|\ldots| \hat{\sigma}_{k}\right] \otimes t / x_{j}+(-1)^{r+1}\left[\check{\sigma}_{1}|\ldots| \check{\sigma}_{k}\right] \otimes t \backslash x_{j}
$$

where $\hat{\sigma}_{i}$ and $\check{\sigma}_{i}$ are the induced permutations of conventional labelling sets for $t / x_{j}$ and $t \backslash x_{j}$. For instance, if $\hat{\sigma}_{k}, \ldots, \hat{\sigma}_{i+1}$ have already been defined, then $\hat{\sigma}_{i}$ is uniquely determined by the stipulation that $\hat{\sigma}_{i} \hat{\sigma}_{i+1} \ldots \hat{\sigma}_{k}\left(t / x_{j}\right)$ be the conventional labelling of $\left(\sigma_{i} \sigma_{i+1} \ldots \sigma_{k} t\right) / x_{j}$. By these means we can define cubical face operators $\varepsilon_{j}$ in $|\mathscr{M}|^{\prime}$ just as in $K_{\infty}$.

In a totally analogous way we can extend the definition of the operators $\Delta_{j}$ to $|\mathscr{M}|^{\prime}$, setting

$$
\Delta_{j}\left(\left[\sigma_{1}|\ldots| \sigma_{k}\right] \otimes t\right)=\left[\bar{\sigma}_{1}|\ldots| \bar{\sigma}_{k}\right] \otimes \Delta_{j}(t),
$$

where $\left[\bar{\sigma}_{1}|\ldots| \bar{\sigma}_{k}\right]$ is the induced string of permutations of the conventional labelling set of $\Delta_{j}(t)$. We define $\Delta$ to be the alternating sum $\sum(-1)^{j} \Delta_{j}$, but we cannot expect this to be a contraction, because of the form of the boundary operator in $|\mathscr{M}|^{\prime}$. Nor is it true that $\bar{\sigma}_{i} \Delta=\Delta \sigma_{i}$, because the action of $\Sigma_{n}$ does not preserve the conventional ordering which is essentially used in the definition of $\Delta$.

In the following definition and all that follows, we use the notation $\bar{\sigma}$ to denote any permutation induced by $\sigma$ on a set of tree labels derived by our conventions. The context always implies exactly what the trees in question are, so it is not necessary to burden the notation with any heavy details.

Definition B.2. We define an operator $\tilde{\Delta}$ on the chains of $|\mathscr{M}|^{\prime}$ by setting

$$
\tilde{\Delta}\left(\left[\sigma_{1}|\ldots| \sigma_{k}\right] \otimes t\right)=\sum_{j=0}^{k}\left\{(-1)^{j}\left[\bar{\sigma}_{1}|\ldots| \bar{\sigma}_{j}\right] \otimes \Delta \bar{\sigma}_{j+1} \Delta \bar{\sigma}_{j+2} \Delta \ldots \Delta \bar{\sigma}_{k} \Delta(t)\right\} .
$$

Theorem B $\cdot 3$. The chain complex $\mathscr{K}_{A}(A ; M)$ is acyclic.

Proof. We have remarked above that it is sufficient to prove that $|\mathscr{M}|^{\prime}$ is contractible, and that we may take $M$ to be $A$. We simply claim that the homotopy $\tilde{\Delta}$, defined above, is a contraction of $|\mathscr{M}|^{\prime}$.

To see this, one repeatedly uses the relation $\partial \Delta+\Delta \partial=1-\pi$ in $K_{\infty}$ to calculate 
that when $t$ is a tree with at least one internal edge, the relation

$$
\begin{aligned}
& \partial\left(\Delta \bar{\sigma}_{j+1} \Delta \bar{\sigma}_{j+2} \Delta \ldots \Delta \bar{\sigma}_{k} \Delta(t)\right) \\
& =\bar{\sigma}_{j+1} \Delta \bar{\sigma}_{j+2} \Delta \ldots \Delta \bar{\sigma}_{k} \Delta(t)+\sum_{r=1}^{k-j-1}(-1)^{r} \Delta \bar{\sigma}_{j+1} \Delta \ldots \Delta \bar{\sigma}_{j+r} \bar{\sigma}_{j+r+1} \Delta \ldots \Delta \bar{\sigma}_{k} \Delta(t) \\
& \quad+(-1)^{k-j} \Delta \bar{\sigma}_{j+1} \Delta \ldots \Delta \sigma_{k} t+(-1)^{k-j-1} \Delta \bar{\sigma}_{j+1} \Delta \ldots \Delta \bar{\sigma}_{k} \Delta(\partial t)
\end{aligned}
$$

holds in $|\mathscr{M}|^{\prime}$, and a minor variant when $t$ is a star-tree. Then straightforward calculation with the formulae defining $\partial$ and $\tilde{\Delta}$ gives

$$
\partial \tilde{\Delta}+\tilde{\Delta} \partial= \begin{cases}1 & \text { in dimension }>0 \\ 1-\pi & \text { in dimension } 0\end{cases}
$$

where $\pi$ is a point map. The theorem is therefore proved.

\section{REFERENCES}

[1] M. André. Méthode simpliciale en algèbre homologique et algèbre commutative. Lect. Notes in Math. (Springer-Verlag, 1967).

[2] M. Barr. Harrison homology, Hochschild homology and triples. J. Algebra 8 (1963), 314-323.

[3] M. Basterra. André-Quillen cohomology of commutative $S$-algebras. J. Pure Appl. Algebra 144 (1999), 111-144.

[4] J. M. BOARDMAN and R. M. VoGT. Homotopy invariant algebraic structures on topological spaces. Lect. Notes in Math. 347 (Springer-Verlag, 1973).

[5] A. K. Bousfield and E. M. Friedlander. Homotopy theory of $\Gamma$-spaces, spectra, and bisimplicial sets. Lect. Notes in Math. 658 (Springer-Verlag, 1978), 80-130.

[6] E. Dyer and R. K. Lashof. Homology of iterated loop spaces. Amer. J. Math. 84 (1962), $35-88$.

[7] E. Getzler and M. M. Kapranov. Cyclic operads and cyclic homology, in Geometry, topology and physics for Raoul Bott (S.-T. Yau, ed.) (Internat. Press, Cambridge, MA., 1995), 167-201.

[8] E. Getzler and M. M. Kapranov. Modular operads. Compositio Math. 110 (1998), 65-126.

[9] P. G. Goenss and M. J. Hopkins. André-Quillen (co-)homology for simplicial algebras over simplicial operads. Contemp. Math. 265 (American Mathematical Society, Providence, RI, $2000), 41-85$.

[10] P. G. Goerss and M. J. Hopkins. Simplicial structured ring spectra (available at http://www.math.nwu.edu/ pgoerss).

[11] P. G. Goerss and M. J. Hopkins. Resolutions in model categories (available at http://www.math.nwu.edu/ pgoerss).

[12] A. Grothendieck. Éléments de géométrie algébrique. Inst. Hautes Études Sci. Publ. Math. $(1960-1967)$.

[13] D. K. Harrison. Commutative algebras and cohomology. Trans. Amer. Math. Soc. 104 (1962), 191-204.

[14] V. Hinich. Homological algebra of homotopy algebras. Comm. Algebra 25 (1997), 3291-3323.

[15] J.-L. Loday. Opérations sur l'homologie cyclique des algèbres commutatives. Invent. Math. 96 (1989), 205-230.

[16] H. R. Miller. A spectral sequence for the homology of an infinite delooping. Pacific J. Math. 79 (1978), 139-155.

[17] T. Pirashvili and B. Richter. Robinson-Whitehouse complex and stable homotopy. Topology 39 (2000), 525-530.

[18] D. G. Quillen. On the (co-)homology of commutative rings, in Proceedings of Symposia in Pure Mathematics (A. Heller, ed.), vol. 17 (American Mathematical Society, Providence, RI, 1970), 65-87.

[19] A. Robinson. Gamma homology, Lie representations and $E_{\infty}$ multiplications (preprint, July 2001).

[20] A. Robinson and S. Whitehouse. The tree representation of $\Sigma_{n+1}$. J. Pure Appl. Algebra 111 (1996), 245-253. 
[21] G. Segal. Categories and cohomology theories. Topology 13 (1974), 293-312.

[22] S. Whitehouse. $\Gamma-(\mathrm{Co})$ homology of commutative algebras and some related representations of the symmetric group. Ph.D. thesis (University of Warwick, 1994).

[23] S. Whitehouse. The integral tree representation of the symmetric group. J. Algebraic Combinatorics 13 (2001), 317-326. 